\title{
TITLE:
}

\section{The Cauchy problem fir hyperbolic operators of strong type}

$\operatorname{AUTHOR}(\mathrm{S})$ :

KAJITANI, KUNIHIKO; NISHITANI, TATSUO; WAKABAYASHI, SEIICHIRO

\section{CITATION:}

KAJITANI, KUNIHIKO ...[et al]. The Cauchy problem fir hyperbolic operators of strong type. 数理解析研究所講究録 1995, 894: 43-66

ISSUE DATE:

1995-01

URL:

http://hdl.handle.net/2433/84438

RIGHT: 
The Cauchy problem for hyperbolic operators of strong type

\author{
Kunihiko KaJitani (梶谷 邦彦)* \\ TATSUO Nishitani (西谷 達雄) ${ }^{* *}$ \\ SEIICHIRO WAKABAYAShI (若林 誠一郎)*
}

\title{
1. Introduction
}

Let $P$ be a differential operator of order $m$ in an open set $\Omega \subset \mathbf{R}^{n}$, and write $P(x, D)=\sum_{j=0}^{m} P_{j}(x, D)$, where the $P_{j}(x, \xi)(0 \leq j \leq m)$ are homogeneous polynomials of $\xi$ of degree $j$ whose coefficients are $C^{\infty}$ functions of $x$ in $\Omega$. Let $z^{0}=\left(x^{0}, \xi^{0}\right) \epsilon$ $T^{*}(\Omega) \backslash 0$ be a characteristic point of order $r$ of the principal part $P_{m}$ of $P$. It is well known that for the Cauchy problem for $P$ to be $C^{\infty}$-well posed the lower order term $P_{j}$ must vanish at least of order $r-2(m-j)$ at $z^{0}$ if $r-2(m-j)>0$, i.e., $j>[(2 m-r) / 2]$, where $[s]$ denotes the integral part of $s$ for $s \in \mathbf{R}$ ( see Ivrii-Petkov [5]). We call the above necessary condition for $C^{\infty}$-well posedness the Ivrii-Petkov condition here. On the other hand it is also known that the Cauchy problem is $C^{\infty}$-well posed for any lower order terms if and only if every characteristic point of $P_{m}$ is at most of order 2 and $P_{m}$ is effectively hyperbolic at every characteristic point of order 2 (see [5], Iwasaki [6] and the references in Nishitani [8]). Therefore, as a generalization of effective.hyperbolicity it is natural to call $P_{m}$ a hyperbolic operator of strong type if the Cauchy problem for $P$ is $C^{\infty}$-well posed for every lower order term $P_{j}$ satisfying the Ivrii-Petkov condition. So a question rises whether there exist hyperbolic operaters of strong type. The answer is positive. Roughly speaking, we can prove that a hyperbolic operator is of strong type if its principal part has the real characteristic roots and the difference of any two roots is bounded from below by the absolute value of a time function, where the precise definition of "time function" will be given later.

In Kajitani-Wakabayashi [9] and [10] we introduced the microlocal a priori estimates to solve the Cauchy problem for linear partial differential operators in $C^{\infty}$, and as its application we investigated the Cauchy problem for such operators with time functions which are in involution. Nishitani studied the same problem for operators

${ }^{*}$ Institute of Mathematics, University of Tsukuba

${ }^{* *}$ Department of Mathematics, College of General Education, Osaka University 
with one time function in [14] and the propagation of singularities in [15]. In this article we shall consider hyperbolic operators of strong type. We shall give a large class of hyperbolic operators of strong type ( see Theorem 1.1 below). Although we have no general results on the characterization of hyperbolic operators of strong type, we believe that our class is very close to the whole class of hyperbolic operators of strong type.

Let $P(x, D)=D_{1}^{m}+\sum_{|\alpha| \leq m, \alpha_{1}<m} a_{\alpha}(x) D^{\alpha}$ be a partial differential operator with coefficients in $C^{\infty}\left(\mathbf{R}^{n}\right)$, where $m$ is a positive integer, $x=\left(x_{1}, x^{\prime}\right)=\left(x_{1}, x_{2}, \cdots, x_{n}\right) \in$ $\mathbf{R}^{n}$ and $D=\left(D_{1}, D^{\prime}\right)=\left(D_{1}, D_{2}, \cdots, D_{n}\right)=-i\left(\partial / \partial x_{1}, \cdots, \partial / \partial x_{n}\right)$. We assume that [A.I] $p(x, \xi)=\xi_{1}^{m}+\sum_{|\alpha|=m, \alpha_{1}<m} a_{\alpha}(x) \xi^{\alpha}$ is hyperbolic with respect to $\theta=(1,0, \cdots$, $0)$, i.e.,

$$
p(x, \xi-i \theta) \neq 0 \quad \text { for } x \in \mathbf{R}^{n} \text { and } \xi \in \mathbf{R}^{n} \text {. }
$$

It is well known that it follows from Lax-Mizohata's theorem [13] that (1.1) is necessary in order that the Cauchy problem for $P(x, D)$ is $C^{\infty}$-well posed.

Let $P(x, \xi)$ be the full symbol of $P(x, D)$ and denote by $P_{j}(x, \xi)$ the homogeneous part of degree $j$ of $P(x, \xi)$. We define $h_{j}(x, \xi)(j=0,1, \cdots, m)$ as $|p(x, \xi-i s \theta)|^{2}=$ $\sum_{j=0}^{m} s^{2(m-j)} h_{j}(x, \xi)$ for $x \in \mathbf{R}^{n}, \xi \in \mathbf{R}^{n}$ and $s \in \mathbf{R}$. Writing $p(x, \xi)=\prod_{j=1}^{m} q_{j}(x, \xi)$, where $q_{j}(x, \xi)=\xi_{1}-\lambda_{j}\left(x, \xi^{\prime}\right)$, we have

$$
h_{j}(x, \xi)=\sum_{1 \leq \ell_{1}<\ell_{2}<\cdots<\ell_{j} \leq m}\left|q_{\ell_{1}}(x, \xi)\right|^{2} \cdots\left|q_{\ell_{j}}(x, \xi)\right|^{2} \quad \text { for } 1 \leq j \leq m
$$

and $h_{0}(x, \xi)=1$. We assume that for $z^{0}=\left(x^{0}, \xi^{0}\right) \in T^{*}\left(\mathbf{R}^{n}\right) \simeq \mathbf{R}^{2 n}$ with $\left|\xi^{0}\right|=1$ $[\mathrm{A} . \mathrm{II}]_{z^{0}}$ there are a conic neighborhood $\mathcal{U}$ of $z^{0}$ and $C>0$ such that

$$
\left|P_{j}(x, \xi)\right| \leq C\left|h_{2 j-m}(x, \xi)\right|^{1 / 2}
$$

for $(x, \xi) \in \mathcal{U}$ with $|\xi|=1$ and $[m / 2]+1 \leq j \leq m-1$.

It should be noted that $h_{2 j-m}(x, \xi) \neq 0$ near $z^{0}$ if $j \leq(2 m-r) / 2$ and $z^{0}$ is a characteristic point of order $r$. We note that there are variants equivalent to the condition $[\mathrm{A} . \mathrm{II}]_{z^{\circ}}$ ( see Lemma 2.5 in [10]). We remark that if the multiple characteristic set $\left\{(x, \xi) \in T^{*}\left(\mathbf{R}^{n}\right) \backslash 0 ; p(x, \xi)=d p(x, \xi)=0\right\}$ of $p(x, \xi)$ is a $C^{\infty}$ manifold, the 
condition $[\mathrm{A} . \mathrm{II}]_{z^{0}}$ is necessary in order that the Cauchy problem for $P(x, D)$ is $C^{\infty}$ well posed (see [5]). We also note that if the coefficients of $P$ belong to a Gevrey class $\mathcal{E}^{(s)}(s \leq 2)$ and the conditions [A.I] and $[\mathrm{A} . \mathrm{II}]_{z^{0}}$ for every $z^{0} \in T^{*}\left(\mathbf{R}^{n}\right)$ with $\left|\xi^{0}\right|=1$ are valid, then the Cauchy problem is well posed in $\mathcal{E}^{(s)}$ ( see Kajitani [8]).

We now introduce the notion of time functions. For a multiple characteristic point $z^{0} \in T^{*}\left(\mathbf{R}^{n}\right) \backslash 0$ of $p$, we denote by $p_{z^{0}}(X)$ the localization of $p$ at $z^{0}$, i.e., $p\left(z^{0}+s X\right)=s^{\mu}\left(p_{z^{0}}(X)+o(1)\right)$ as $s \rightarrow 0$ and $p_{z^{0}}(X) \not \equiv 0$ in $X \in T_{z^{0}}\left(T^{*} \mathbf{R}^{n}\right)$. Denote by $\Gamma\left(p_{z^{0}},(0, \theta)\right)$ the connected component of the set $\left\{X \in T_{z^{0}}\left(T^{*}\left(\mathbf{R}^{n}\right)\right) ; p_{z^{0}}(X) \neq 0\right\}$ containing $(0, \theta)$. We say that $t(x, \xi)$ is a time function of $p$ at $z^{0}$ if $t(x, \xi)$ is real-valued, continuous in $T^{*}\left(\mathbf{R}^{n}\right) \backslash 0$ and positively homogeneous in $\xi$ of degree 0 and if there are a conic neighborhood $\mathcal{U}$ of $z^{0}$ and a compact convex set $K \subset \Gamma\left(p_{z^{0}},(0, \theta)\right)$ such that $t(x, \xi)$ is Lipschitz continuous in $\mathcal{U} \cap\{1 / 2 \leq|\xi| \leq 2\}$ and $-\left(|\xi| \nabla_{\xi} t(x, \xi),-\nabla_{x} t(x, \xi)\right) \in K$ for a.e. $(x, \xi) \in \mathcal{U}$. In particular, $t(x, \xi)$ is a time function of $p$ at $z^{0}$, if $t(x, \xi)$ is a real valued function in $C^{1}\left(T^{*}\left(\mathbf{R}^{n}\right) \backslash 0\right)$ and positively homogenuous of degree 0 and $-H_{t}\left(z^{0}\right) \equiv-\left(\nabla_{\xi} t,-\nabla_{x} t\right)\left(z^{0}\right) \in \Gamma\left(p_{z^{0}},(0, \theta)\right)$. In fact, it follows from continuity in $z$ of $-H_{t}(z)$ that there are a conic neighborhood $\mathcal{U}$ of $z^{0}$ and a compact convex set $K \subset \Gamma\left(p_{z^{0}},(0, \theta)\right)$ such that $-H_{t}(z) \in K$ for $z \in \mathcal{U}$.

For a multiple characteristic point $z^{0}=\left(x^{0}, \xi^{0}\right) \in T^{*}\left(\mathbf{R}^{n}\right)$ with $\left|\xi^{0}\right|=1$ we assume that

$[\mathrm{A} . \mathrm{III}]_{z^{0}}$ there are a conic neighborhood $\mathcal{U}$ of $z^{0}$ and time functions $t_{\ell}(x, \xi)(1 \leq \ell \leq d)$ of $p$ at $z^{0}$ such that

$$
h_{m-1}(x, \xi) \geq h_{m-2}(x, \xi) t(x, \xi)^{2} \quad \text { for }(x, \xi) \in \mathcal{U} \text { with }|\xi|=1
$$

where $t(x, \xi)=\min _{1 \leq \ell \leq d}\left|t_{\ell}(x, \xi)\right|$.

In particular, $[\mathrm{A} . \mathrm{III}]_{z^{0}}$ implies that the multiple characteristic set of $p$ is included in the union of the zeros of finitely many time functions. We note that the condition (1.2) is equivalent to

$$
\left|q_{j}(x, \xi)\right|+\left|q_{k}(x, \xi)\right| \geq c t(x, \xi) \quad \text { for }(x, \xi) \in \mathcal{U} \text { with }|\xi|=1 \text { and } j \neq k
$$

where $p(x, \xi)=\prod_{j=1}^{m} q_{j}(x, \xi)$ and $c>0$ ( see Lemma 2.4 in [10]). Since it follows from [1] that the $q_{j}$ are Lipschitz continuous in $T^{*}\left(\mathbf{R}^{n}\right) \backslash 0,(1.3)$ is valid if there are $c_{j k} \in \mathbf{R}$ such that $\left(c_{j k} q_{j}+c_{k j} q_{k}\right)|\xi|^{-1}$ are time functions of $p$ at $z^{0}$ for $j \neq k$. 
Now we can state our main result. Let $\Omega$ be a bounded domain in $\mathbf{R}^{n}$ containing the origin. Under the assumption [A.I] we can define the generalized flows issuing from $x^{0}$ in $\mathbf{R}^{n}$ by

$$
\begin{aligned}
K_{x^{0}}^{ \pm}= & \left\{x(t) ; \pm t \geq 0, \text { and }\{x(t)\} \text { is a Lipschitz continuous curve in } \mathbf{R}^{n}\right. \\
& \text { satisfying } \left.d x(t) / d t \in \Gamma(p(x(t), \cdot), \theta)^{*}(\text { a.e. } t) \text { and } x(0)=x^{0}\right\}
\end{aligned}
$$

where $\Gamma(p(x, \cdot), \theta)$ denotes the connected component of the set $\left\{\xi \in \mathbf{R}^{n} ; p(x, \xi) \neq 0\right\}$ containing $\theta$ and $\Gamma^{*}=\left\{x \in \mathbf{R}^{n} ; x \cdot \xi \geq 0\right.$ for any $\left.\xi \in \Gamma\right\}$.

Theorem 1.1. Assume that the conditions [A.I] is satisfied and that [A.II] $z^{0}$ and $[\mathrm{A} . \mathrm{III}]_{z^{0}}$ are satisfied for $z^{0}=\left(x^{0}, \xi^{0}\right) \in T^{*}\left(\mathbf{R}^{n}\right)$ with $|\xi|=1, x^{0} \in \bar{\Omega}, x_{1}^{0} \geq 0$ and $d p\left(z^{0}\right)=0$. Then for any $f \in \mathcal{D}^{\prime}$ with supp $f \subset\left\{x_{1} \geq 0\right\}$ the Cauchy problem

$$
\left\{\begin{array}{l}
P(x, D) u(x)=f \quad \text { in } \Omega, \\
\text { supp } u \subset\left\{x_{1} \geq 0\right\}
\end{array}\right.
$$

has a solution $u \in \mathcal{D}^{\prime}$. If $x^{0} \in \Omega, K_{x^{0}}^{-} \cap\left\{x_{1} \geq 0\right\} \subset \Omega$, and $u \in \mathcal{D}^{\prime}$ satisfies (CP) with $f=0$ near $K_{x^{0}}^{-}$, then $x^{0} \notin \operatorname{supp} u$. Moreover if $x^{0} \in \Omega, K_{x^{0}}^{-} \cap\left\{x_{1} \geq 0\right\} \subset \Omega, u \in \mathcal{D}^{\prime}$ satisfies (CP) and $f \in C^{\infty}\left(\mathbf{R}^{n}\right)$, then $x^{0} \notin \operatorname{sing} \operatorname{supp} u$, i.e., $u \in C^{\infty}$ near $x^{0}$.

Here we give a simple example to elucidate the geometrical meanings of the conditions $[\mathrm{A} . \mathrm{II}]_{z^{0}}$ and $[\mathrm{A} . \mathrm{III}]_{z^{0}}$. Let $p(x, \xi)$ be factorized as

$$
p(x, \xi)=e(x, \xi) \prod_{j=1}^{r} q_{j}(x, \xi)
$$

in a conic neighborhood $\mathcal{U}$ of $z^{0}$, where $e(x, \xi)$ and $q_{j}(x, \xi)$ are smooth near $z^{0}$ and positively homogeneous of degree $m-r$ and 1 , respectively, and $e\left(z^{0}\right) \neq 0, d q_{j}\left(z^{0}\right) \neq$ 0 and $q_{j}\left(x^{0}, \theta\right)>0$. Assume, for simplicity, that $d q_{j}$ are linearly independent at $z^{0}$. Recall that the cone generated by the Hamilton vector fields $H_{q_{j}}\left(z^{0}\right)$ of $q_{j}$ forms the propagation cone $\Gamma\left(p_{z^{0}},(0, \theta)\right)^{\sigma}$ of the localization $p_{z^{0}}(X)$, where $\Gamma^{\sigma}=\{X \in$ $T_{z^{0}}\left(T^{*}\left(\mathbf{R}^{n}\right)\right) ; \sigma(Y, X) \geq 0$ for any $\left.Y \in \Gamma\right\}$ for $\Gamma \subset T_{z^{0}}\left(T^{*}\left(\mathbf{R}^{n}\right)\right)$ and $\sigma=\sum d \xi_{j} \wedge d x_{j}$. Then the condition [A.III $]_{z^{0}}$ is fulfilled if and only if $\Gamma\left(p_{z^{0}},(0, \theta)\right)^{\sigma}$ is transversal to the tangent space at $z^{0}$ of each intersection of any two hypersurfaces $q_{k}=0, q_{j}=0$ (see [15]). On the other hand, the condition [A.II] $z^{0}$ is satisfied if and only if $P_{j}(x, \xi)$ vanishes of order $r-2(m-j)$ on each intersection of any two hypersurfaces $q_{k}=0$, 
$q_{\ell}=0$ near $z^{0}$ whenever $r-2(m-j)>0$. Even if the $q_{j}(x, \xi)$ are not smooth in (1.4), we can show that (1.2) implies that

$$
\Gamma\left(p_{z^{0}},(0, \theta)\right)^{\sigma} \cap \Sigma\left(p_{z^{0}}\right)=\{0\}
$$

where $\Sigma\left(p_{z^{0}}\right)=\left\{X \in T_{z^{0}}\left(T^{*}\left(\mathbf{R}^{n}\right)\right) ; d p_{z^{0}}(X)=0\right\}$. If $z^{0}$ is a characteristic point of order 2 , or if $p(x, \xi)$ can be smoothly factorized as (1.4), then the converse is also true ( see [8]). So one can conjecture that the condition (1.5) is necessary for (CP) to be $C^{\infty}$-well posed with arbitrary lower order terms $P_{j}$ satisfying $[\mathrm{A} . \mathrm{II}]_{z^{0}}$.

The major part of the proof of Theorem 1.1 is devoted to the proof of Theorem 2.2 below. We now explain the basic ideas proving the microlocal a priori estimates $(2.1)_{z^{0}}$ in Theorem 2.2 for a fixed $z^{0}$. To derive the microlocal a priori estimates we consider $P_{\Lambda}(x, D)=e^{\Lambda}(x, D)^{-1} P(x, D) e^{\Lambda}(x, D)$, where $e^{\Lambda}(x, D)$ is a pseudodifferential operator with symbol $e^{\Lambda(x, \xi)}$. Here the Hamilton vector field $H_{\Lambda}$ of $\Lambda(x, \xi)$ satisfies

$$
H_{\Lambda} \sim M \sum_{j=1}^{d}\left(t_{j}(x, \xi)^{2}+N|\xi|^{-1}\right)^{-1 / 2} H_{t_{j}}
$$

where $M$ and $N$ are large parameters and the $t_{j}(x, \xi)$ are the time functions in $[\mathrm{A} . \mathrm{III}]_{z^{0}}$. The conjugated operator $P_{\Lambda}(x, D)$ admits the following expansion:

$$
P_{\Lambda}(x, \xi) \sim \sum_{j=0}^{m}\left(i H_{\Lambda}\right)^{j} p(x, \xi) / j !+\cdots
$$

To estimate the norm of $P_{\Lambda} u$ from below, following the classical idea attributed to Leray [12] and Gårding [3] we separate $P_{\Lambda}$ by $Q(x, D)$ and estimate

$$
\operatorname{Im}\left(P_{\Lambda}(x, D) u, Q(x, D) u\right)_{L^{2}}=(S(x, D) u, u)_{L^{2}}
$$

where $S(x, D)=(2 i)^{-1}\left(Q^{*} P_{\Lambda}-P_{\Lambda}^{*} Q\right)$ and $(f, g)_{L^{2}}$ denotes the inner product of $L^{2}\left(\mathbf{R}^{n}\right)$. A desired $Q(x, \xi)$ is given by

$$
Q(x, \xi)=|\widetilde{H}|^{-1} \widetilde{H} \sum_{j=0}^{m}\left(i H_{\Lambda}\right)^{j} p(x, \xi) / j !
$$

where $\widetilde{H}=\left(\langle\xi\rangle_{h} \nabla_{\xi} \Lambda(x, \xi),-\nabla_{x} \Lambda(x, \xi)\right)$ and $\langle\xi\rangle_{h}=\left(h^{2}+|\xi|^{2}\right)^{1 / 2}$. From the conditions $[\mathrm{A} . \mathrm{II}]_{z^{0}}$ and $[\mathrm{A} . \mathrm{III}]_{z^{0}}$ and microhyperbolicity for $p(x, \xi)$ with respect to $-H_{t_{j}}$ ( see 
[16]), $S(x, \xi)$ becomes a "weight" in the sense of Hörmander [4] and $S(x, \xi)-S_{0}(x, \xi) \in$ $S\left(N M^{-2} S_{0}, g\right)$, where a suitable metric $g$ associates with the $t_{j}(x, \xi)$ and $S_{0}(x, \xi)$ is the principal part of $S(x, \xi)$. Then, if we can choose $N M^{-2} \ll 1$, constructing an inverse of $S(x, D)$, we can show that $(1.6)$ is bounded from below by $\|u\|_{H^{(m-1) / 2}\left(\mathbf{R}^{n}\right)}^{2}$. Then $P_{\Lambda}$ satisfies the microlocal a priori estimates and, therefore, $P$ also does. From the microlocal a priori estimates we can prove Theorem 1.1, following the idea of the proof of Theorem 1.5 in [9].

In our argument, under the restriction that $N M^{-2} \ll 1$, we must construct an inverse of the pseudodifferential operator with symbol

$$
e^{\Lambda(x, \xi)} \sim \prod_{j=1}^{d}\left(\left(t_{j}(x, \xi)^{2}|\xi|+N\right)^{1 / 2}+t_{j}(x, \xi)|\xi|^{1 / 2}\right)^{M}
$$

In [10] we consider the cases where the $t_{j}(x, \xi)$ are in involution. If the $t_{j}(x, \xi)$ are not in involution, we have serious difficulties in this construction, since $e^{\Lambda}(x, D)$ is a pseudodifferential operator of type $1 / 2,1 / 2$ with two large parameters. This construction is essential in proving Theorem 1.1, which will be given in $\S 3$.

\section{Proof of Theorem 1.1}

In this section we shall give an outline of the proof of Theorem 1.1 from the microlocal a priori estimates ( see Theorem 2.2 below).

Let $\Omega$ be a bounded domain in $\mathbf{R}^{n}$. Denote $\Omega_{+}=\Omega \cap\left\{x_{1}>0\right\}$ and $\Omega_{+, \delta}=\{x \in$ $\left.\mathbf{R}^{n} ; \operatorname{dis}\left(x, \Omega_{+}\right)<\delta\right\}$ for $\delta>0$. Let $\delta>0$ and choose $R>0$ so that $\Omega_{+, 2 \delta} \subset\{|x|<R\}$. Moreover, choose $\varphi_{\delta}(x) \in C^{\infty}\left(\mathbf{R}^{n}\right)$ and $\chi_{R, \delta}(x) \in C^{\infty}\left(\mathbf{R}^{n} ; \mathbf{R}^{n}\right)$ such that $\varphi_{\delta}(x)=1$ in $\Omega_{+, \delta}$ and $\varphi_{\delta}=0$ in the complement of $\Omega_{+, 2 \delta}$ and $\chi_{R, \delta}(x)=x$ in $\{|x|<R\}$ and $=R x /|x|$ in $\{|x| \geq R+\delta\}$. For $P(x, \xi)=\sum_{|\alpha| \leq m} a_{\alpha}(x) \xi^{\alpha}$ we define

$$
\begin{aligned}
\widetilde{P}(x, \xi)= & \left(1-\left(1-\varphi_{\delta}(x)\right)^{2}\left|\xi^{\prime}\right|^{2}\left(\partial / \partial \xi_{1}\right)^{2}\right)^{m-1} \sum_{|\alpha|=m} a_{\alpha}\left(\chi_{R, \delta}(x)\right) \xi^{\alpha} \\
& +\varphi_{\delta / 2}(x) \sum_{|\alpha|<m} a_{\alpha}(x) \xi^{\alpha}
\end{aligned}
$$

We denote by $\widetilde{p}(x, \xi)$ the principal part of $\widetilde{P}$. Then we have the following

Proposition 2.1. Assume that $P(x, \xi)$ satisfies [A.I], [A.II $]_{z^{0}}$ and $[\mathrm{A} . \mathrm{III}]_{z^{0}}$. Then, (i) $\widetilde{P}(x, \xi)=P(x, \xi)$ for $x \in \Omega_{+, \delta / 2}$, (ii) the coefficients of $\widetilde{P}$ belong to $\mathcal{B}^{\infty}\left(\mathbf{R}^{n}\right)$ 
and $\widetilde{p}(x, \xi)$ is strictly hyperbolic with respect to $\theta=(1,0, \cdots, 0)$ in the complement of $\Omega_{+, 2 \delta}$, and (iii) $\widetilde{P}(x, \xi)$ satisfies $[\mathrm{A} . \mathrm{I}],[\mathrm{A} . \mathrm{II}]_{z^{0}}$ and $[\mathrm{A} . \mathrm{III}]_{z^{0}}$.

Let $z^{0}=\left(x^{0}, \xi^{0}\right) \in T^{*}\left(\mathbf{R}^{n}\right)$ be fixed so that $\left|\xi^{0}\right|=1$ and $d p\left(z^{0}\right)=0$. We use the following notations: (i) $P_{\gamma}(x, D)=P(x, D-i \gamma \theta)$ for $\gamma>0$ and $\theta=(1,0, \cdots, 0) \in$ $\mathbf{R}^{n}$, (ii) $t_{ \pm}(x, \xi) \in \mathcal{B}^{\infty}\left(T^{*}\left(\mathbf{R}^{n}\right) \backslash 0\right)$, positively homogeneous in $\xi$ of degree 0 , satisfying $t_{ \pm}(x, \xi)= \pm\left(x_{1}-x_{1}^{0}\right)+\left|x-x^{0}\right|^{2}+|\xi /| \xi\left|-\xi^{0}\right|^{2}$ in a conic neighborhood of $z^{0}$, (iii) $\Theta(t) \in C_{0}^{\infty}(\mathbf{R})$ satisfying $0 \leq \Theta(t) \leq 1$ and $\Theta(t)=1$ for $|t| \leq 1$ and $\Theta(t)=0$ for $|t| \geq 2$, (iv) $\Theta_{h}(\xi)=\Theta(|\xi| / h)$ for $h>0$, (v) $\varphi_{i}(x, \xi) \in C^{\infty}\left(T^{*}\left(\mathbf{R}^{n}\right) \backslash 0\right)$ ( $i=1,2)$ being positively homogeneous in $\xi$ of degree 0 , (vi) $\varphi_{i, h}(x, \xi)=(1-$ $\left.\Theta_{h / 2}(\xi)\right) \varphi_{i}(x, \xi),($ vii $) \zeta(x)$ satisfying $\nabla \zeta(x) \in \mathcal{B}^{\infty}\left(\mathbf{R}^{n}\right)$ and $\zeta(x)=\theta^{0} \cdot\left(x-x^{0}\right)+k \mid x-$ $\left.x^{0}\right|^{2}$ near $x^{0}$ for fixed $x^{0} \in \mathbf{R}^{n}, k>0$ and $\theta^{0} \in \Gamma\left(p\left(x^{0}, \cdot\right), \theta\right)$, (viii) $P(x, D ; \gamma) u(x)=$ $e^{-\gamma \zeta(x)} P(x, D)\left(e^{\gamma \zeta(x)} u(x)\right)$, (ix) $\langle\xi\rangle_{h}=\left(h^{2}+|\xi|^{2}\right)^{1 / 2}$ for $h>0,(\mathbf{x}) \Lambda_{ \pm}(x, \xi) \equiv$ $\Lambda_{ \pm, a, b}(x, \xi)=\left\{\left(a t_{ \pm}(x, \xi)-b\right) \log \langle\xi\rangle_{h}\right\} \varphi_{2, h / 2}(x, \xi)$ for $a \geq 1$ and $b \in \mathbf{R}$, and (xi) $P_{\gamma \Lambda_{ \pm}}(x, D)=e^{-\Lambda_{ \pm}}(x, D) P_{\gamma}(x, D) e^{\Lambda_{ \pm}}(x, D), P_{\Lambda_{ \pm}}(x, D ; \gamma)=e^{-\Lambda_{ \pm}}(x, D) P(x, D ; \gamma)$ $\times e^{\Lambda_{ \pm}}(x, D)$, where $e^{\Lambda_{ \pm}}(x, D)$ are pseudodifferential operators with symbols $e^{\Lambda_{ \pm}(x, \xi)}$. Similarly, we define $\widetilde{P}(x, D ; \gamma), \cdots$ and so on.

We begin with the microlocal a priori estimates for $\widetilde{P}$ at $z^{0}$.

Theorem 2.2. Let $z^{0}=\left(x^{0}, \xi^{0}\right)$ with $\left|\xi^{0}\right|=1$ be a multiple characteristic point of $p$. Assume that $P(x, D)$ satisfies [A.I], $[\mathrm{A} . \mathrm{II}]_{z^{0}}$ and $[\mathrm{A} . \mathrm{III}]_{z^{\mathrm{o}}}$. Then there are conic neighborhoods $\mathcal{U}_{1}$ and $\mathcal{U}_{2}$ of $z^{0}, \varphi_{i}(x, \xi) \in C^{\infty}\left(T^{*}\left(\mathbf{R}^{n}\right) \backslash 0\right)(i=1,2)$ and $\ell_{i} \in \mathbf{R}$ $(i=1,2,3)$ such that $\operatorname{supp} \varphi_{2} \subset \mathcal{U}_{2}, \varphi_{i}(x, \xi)=1$ in $\mathcal{U}_{1}$ and for any $a \geq 1$ and $b \in \mathbf{R}$ there are $\gamma_{0} \geq 1$ and $C>0$ satisfying

$$
\left\|\langle D\rangle_{h}^{\ell_{1}} v\right\|_{L^{2}} \leq C\left\{\left\|\langle D\rangle_{\gamma}^{\ell_{2}} \widetilde{P}_{\Lambda_{+}}(x, D ; \gamma) v\right\|_{L^{2}}+\left\|\langle D\rangle_{\gamma}^{\ell_{3}}\left(1-\varphi_{1, h}(x, D)\right) v\right\|_{L^{2}}\right\}
$$

if $v \in C_{0}^{\infty}\left(\mathbf{R}^{n}\right)$ and $h=\gamma \geq \gamma_{0}$.

An outline of the proof of this theorem will be given in $\S 4$. The inequality $(2.1)_{z^{0}}$ is called the microlocal a priori estimate for $\widetilde{P}$. We can also obtain the microlocal a priori estimates with $\Lambda_{+}$replaced by $\Lambda_{-}$for ${ }^{t} \widetilde{P}$. From Theorem 2.2 we have the following

Lemma 2.3 ( Lemma 3.1 in [9]). There is $\varphi(x) \in C_{0}^{\infty}\left(\mathbf{R}^{n}\right)$ such that $\varphi(x)=1$ near $x^{0}$ and for any $s \geq 0$ there are $\ell^{\prime}, \ell^{\prime \prime} \in \mathbf{R}$ satisfying the following; for any $\ell \in \mathbf{R}$ 
there are $\gamma_{0} \geq 1$ and $C>0$ such that

$$
\begin{aligned}
\left\|\langle D\rangle_{\gamma}^{\ell} \varphi(x) u\right\|_{L^{2}} \leq C\left\{\|\langle D\rangle_{\gamma}^{\ell+\ell^{\prime}} \chi\left(x_{1}\right)\right. & \widetilde{P}(x, D ; \gamma) u \|_{L^{2}} \\
& \left.+\left\|\langle D\rangle_{\gamma}^{\ell-s} u\right\|_{L^{2}}+\left\|\langle D\rangle_{\gamma}^{\ell+\ell^{\prime \prime}} \chi\left(x_{1}\right) \Theta_{2 h}(D) u\right\|_{L^{2}}\right\}
\end{aligned}
$$

if $u \in C_{0}^{\infty}\left(\mathbf{R}^{n}\right)$, supp $u \subset\left\{x_{1}>x_{1}^{0}-\delta_{0}\right\}$ and $h=\gamma \geq \gamma_{0}$, where $\chi\left(x_{1}\right)=\Theta\left(\left(x_{1}-\right.\right.$ $\left.\left.x_{1}^{0}\right) /\left(3 \delta_{0}\right)\right)$.

Let $\Omega_{0}$ be a bounded domain in $\mathbf{R}^{n}$ such that $\nabla \zeta(x) \in \Gamma(\tilde{p}(x, \cdot), \theta)$ for $x \in \bar{\Omega}_{0}$. Using ellipticity of $\widetilde{P}(x, \xi ; \gamma)$ for $x \in \bar{\Omega}_{0}$ and $\xi \in \mathbf{R}^{n}$ with $|\xi| \leq C \gamma$, we have the following

Lemma 2.4. For any $t \in \mathbf{R}$ and $\delta_{0}>0$ there is $\delta_{0}^{\prime}>0$ such that for any $s \geq 0$ there is $\ell^{\prime} \in \mathbf{R}$ satisfying the following; for any $\ell \in \mathbf{R}$ there are $\gamma_{0} \geq 1$ and $C>0$ such that

$$
\left\|\langle D\rangle_{\gamma}^{\ell} \tau_{\delta_{0}^{\prime}}\left(x_{1}-t\right) u\right\|_{L^{2}} \leq C\left\{\left\|\langle D\rangle_{\gamma}^{\ell+\ell^{\prime}} \tau_{\delta_{0}}\left(x_{1}-t\right) \tilde{P}(x, D ; \gamma) u\right\|_{L^{2}}+\left\|\langle D\rangle_{\gamma}^{\ell-s} u\right\|_{L^{2}}\right\}
$$

if $u \in C_{0}^{\infty}\left(\Omega_{0} \cap\left\{x_{1}>t-\delta_{0}^{\prime}\right\}\right)$ and $\gamma \geq \gamma_{0}$, where $\tau_{\delta^{\prime}}\left(x_{1}\right)=\Theta\left(2 x_{1} / \delta^{\prime}\right)$.

In particular, Lemma 2.4 gives a Carleman estimate for $\widetilde{P}(x, D)$. Therefore, we have the following

Theorem 2.5 ( Theorem 1.2 in [9]). Assume that $P$ satisfies [A.I], [A.II] $]_{z^{0}}$ and [A.III $]_{z^{0}}$ for any multiple characteristic point $z^{0}$ of $p$. Then for any $x^{0} \in \mathbf{R}^{n}$ there is a neighborhood $U_{0}$ of $x^{0}$ such that $x^{0} \notin \operatorname{supp} u$ if $u \in \mathcal{D}^{\prime}$, supp $\widetilde{P} u \cap \bar{V}=\emptyset$ and supp $u \cap\left\{x \in \partial V ; \zeta(x) \leq \zeta\left(x^{0}\right)\right\}=\emptyset$ for some neighborhood $V$ of $x^{0}$ with $V \subset U_{0}$.

The method of sweeping out due to John [7] proves the following uniqueness theorem, noting that $\widetilde{K}_{x}^{-} \cap\left\{x_{1} \geq t\right\}$ is bounded for each $x \in \mathbf{R}^{n}$ and $t \in \mathbf{R}$, where $\widetilde{K}_{x}^{ \pm}$ denote the generalized flows issuing from $x$ for $\widetilde{P}$ ( see, e.g., [12], [16] and [17]).

Theorem 2.6. Assume that the hypotheses of Theorem 2.5 are fulfilled. Let $x^{0} \in \mathbf{R}^{n}$ and $t \in \mathbf{R}$. Then $x^{0} \notin \operatorname{supp} u$ if $u \in \mathcal{D}^{\prime}$, supp $u \subset\left\{x_{1} \geq t\right\}$ and $\widetilde{P} u=0$ near $\widetilde{K}_{x^{0}}^{-}$.

Note that $-\theta \in \Gamma(\tilde{p}(x, \cdot),-\theta)$ for $x \in \mathbf{R}^{n}$. By Theorem 2.2 we obtain the microlocal a priori estimates with $\Lambda_{+}$replaced by $\Lambda_{-}$for ${ }^{t} \tilde{P}$. Applying the same argument as in the proof of Lemma 2.4, we can see that for any $t \in \mathbf{R}$ and $\delta_{0}>0$ there is $\delta_{0}^{\prime}>0$ 
such that for any $s \geq 0$ there is $\ell^{\prime} \in \mathbf{R}$ satisfying the following; for any $\ell \in \mathbf{R}$ there are $\gamma_{0} \geq 1$ and $C>0$ such that

$$
\begin{aligned}
& \left\|\langle D\rangle_{\gamma}^{\ell} \tau_{\delta_{0}^{\prime}}\left(x_{1}-t\right) u\right\|_{L^{2}} \\
& \leq C\left\{\left\|\langle D\rangle_{\gamma}^{\ell+\ell^{\prime}} \tau_{\delta_{0}}\left(x_{1}-t\right)^{t} \widetilde{P}(x, D+i \gamma \theta) u\right\|_{L^{2}}+\left\|\langle D\rangle_{\gamma}^{\ell-s} u\right\|_{L^{2}}\right\}
\end{aligned}
$$

if $u \in C_{0}^{\infty}\left(\Omega_{0} \cap\left\{x_{1}<t+\delta_{0}^{\prime}\right\}\right)$ and $\gamma \geq \gamma_{0}$. Define

$$
\|u\|_{+, \gamma,(m, s)}=\inf \left\{\left\|\langle D\rangle_{\gamma}^{m}\left\langle D^{\prime}\right\rangle_{\gamma}^{s} U\right\| ;\left.U\right|_{X}=u \text { and } U \in \mathcal{H}_{(m, s)}\left(\mathbf{R}^{n}\right)\right\}
$$

where $X=\left\{x \in \mathbf{R}^{n} ; x_{1}>-6 \delta\right\}$. By the same argument as in [9] we can see that there is $\ell^{\prime} \in \mathbf{R}$ such that for any $\ell \in \mathbf{R}$ there are $\gamma_{0} \geq 1$ and $C>0$ satisfying

$$
\|u\|_{+, \gamma,(\ell, 0)} \leq C\left\{\left\|^{t} \widetilde{P}(x, D+i \gamma \theta) u\right\|_{+, \gamma,\left(\ell+\ell^{\prime}, 0\right)}+\left\|{ }^{t} \widetilde{P}(x, D+i \gamma \theta) u\right\|_{+, \gamma,\left(0, \ell+\ell^{\prime}\right)}\right\}
$$

if $u \in C_{0}^{\infty}\left(\Omega_{0}\right)$ and $\gamma \geq \gamma_{0}$. By the Hahn-Banach theorem and Theorem 2.6 we can prove Theorem 1.1. For the detail of the proof we refer to [11].

\section{Construction of the inverse of $e^{\Lambda}(x, D)$}

Let $t_{k}(x, \xi)(1 \leq k \leq d)$ be real valued bounded mesurable functions in $\mathbf{R}^{2 n}$ satisfying

$$
\left|t_{k}(x+y, \xi+\eta)-t_{k}(x, \xi)\right| \leq C\left\{|y|+|\xi|^{-1}|\eta|\right\}
$$

for $(x, \xi),(y, \eta) \in \mathbf{R}^{2 n}$ and $|\eta| \leq|\xi| / 2$. Put $t_{k, h}(x, \xi)=\left(1-\Theta_{h}(\xi)\right) t_{k}(x, \xi)(1 \leq k \leq d)$, where $\Theta_{h}(\xi)$ was given in $\S 2$. Then $t_{k, h}$ satsifies

$$
\left|t_{k, h}(x+y, \xi+\eta)-t_{k, h}(x, \xi)\right| \leq C\left\{|y|+\langle\xi\rangle_{h}^{-1}|\eta|\right\}
$$

for $(x, \xi),(y, \eta) \in \mathbf{R}^{2 n}$, where $\langle\xi\rangle_{h}=\left(h^{2}+|\xi|^{2}\right)^{1 / 2}$. Put, with parameters $h \geq 1$ and $N \geq d$

$$
\begin{gathered}
n_{k}(x, \xi)=\left\{t_{k, h}(x, \xi)^{2}\langle\xi\rangle_{h}+N\right\}^{1 / 2}, \quad T_{k}(x, \xi)=n_{k}(x, \xi)+t_{k, h}(x, \xi)\langle\xi\rangle_{h}^{1 / 2} \\
\varphi_{k}(x, \xi)=\langle\xi\rangle_{h}^{-1 / 2} n_{k}(x, \xi), \quad \Psi_{k}(x, \xi)=\varphi_{k}(x, \xi)\langle\xi\rangle_{h} \\
\varphi(x, \xi)^{-1}=\sum_{k=1}^{d} \varphi_{k}(x, \xi)^{-1}, \quad \Psi(x, \xi)=\varphi(x, \xi)\langle\xi\rangle_{h} \\
g_{(x, \xi)}(y, \eta)=\varphi(x, \xi)^{-2}|y|^{2}+\Psi(x, \xi)^{-2}|\eta|^{2} \\
g_{(x, \xi)}^{\sigma}(y, \eta)=\Psi(x, \xi)^{2}|y|^{2}+\varphi(x, \xi)^{2}|\eta|^{2}
\end{gathered}
$$


Following [4] we say that a positive real valued function $m(x, \xi)$ defined in $\mathbf{R}^{2 n}$ is $g$ continuous, if there are positive constants $c_{0}$ and $C$ such that if $g_{(x, \xi)}(y, \eta) \leq c_{0}$,

$$
C^{-1} m(x, \xi) \leq m(x+y, \xi+\eta) \leq C m(x, \xi)
$$

and that a $g$ continuous function $m(x, \xi)$ is $\sigma, g$ temperate, if there are $C>0$ and $\ell \in \mathbf{R}$ such that

$$
m(x+y, \xi+\eta) \leq C m(x, \xi)\left(1+g_{(x, \xi)}^{\sigma}(y, \eta)\right)^{\ell}
$$

for $(x, \xi),(y, \eta) \in \mathbf{R}^{2 n}$. For simplicity we denote $A \sim B$ if $A / B$ and $B / A$ are bounded. For example $m(x+y, \xi+\eta) \sim m(x, \xi)$ means (3.2). By giving a series of lemmas without proof we want to show an outline of the construction of the inverse of $e^{\Lambda}(x, D)$. For the detail of the proof we refer to [11].

Lemma 3.1. Assume that (3.1) is valid and $h \geq N \geq d$. Then $\langle\xi\rangle_{h}$ and $n_{k}(x, \xi)$ are $\sigma, g$ temperate.

It follows from Lemma 3.1 that $\varphi_{k}, \Psi_{k}, \varphi$ and $\Psi$ are $\sigma, g$ temperate. Hence we have the following

Lemma 3.2. Assume that (3.1) is valid and $h \geq N \geq d$. Then the metric $g_{(x, \xi)}(y, \eta)$ is $\sigma$ temperate.

Lemma 3.3. Assume that (3.1) is valid and $h \geq N \geq d$. Then, (i)

$$
\varphi(x+y, \xi+\eta)^{-1} \leq C \varphi(x, \xi)^{-1}\left\{1+N^{-2} g_{(x, \xi)}^{\sigma}(y, \eta)\right\}^{1 / 4}
$$

for $(x, \xi),(y, \eta) \in \mathbf{R}^{2 n}$. (ii)

$$
\varphi(x+y, \xi+\eta) \leq C \varphi(x, \xi)\left\{1+N^{-2} g_{(x, \xi)}^{\sigma}(y, \eta)\right\}^{1 / 2}
$$

for $(x, \xi),(y, \eta) \in \mathbf{R}^{2 n}$. (iii) $\Psi$ also satisfies (i) and (ii), replacing $\varphi$ by $\Psi$.

Lemma 3.4. Assume that (3.1) is valid and $h \geq N \geq d$. Then $T_{k}(x, \xi)=$ $n_{k}(x, \xi)+t_{k, h}(x, \xi)\langle\xi\rangle_{h}^{1 / 2}(1 \leq k \leq d)$ are $\sigma, g$ temperate and satisfy

$$
T_{k}(x+y, \xi+\eta)^{ \pm 1} \leq C T_{k}(x, \xi)^{ \pm 1}\left(1+N^{-2} g_{(x, \xi)}^{\sigma}(y, \eta)\right)
$$

for $(x, \xi),(y, \eta) \in \mathbf{R}^{2 n}$. 
We have to approximate $\varphi$ and $\Psi$ by functions in some Gevrey class. Let $\rho(z)$ be a function in $C_{0}^{\infty}\left(\mathbf{R}^{2 n}\right)$ satisfying $\rho(z) \geq 0, \rho(z)=0$ for $|z| \geq c_{0}, \int \rho(z) d z=1$ and

$$
\left|D_{z}^{\alpha} \rho(z)\right| \leq C A^{|\alpha|}|\alpha| !^{\kappa} \quad \text { for } z \in \mathbf{R}^{2 n}
$$

where $\kappa>1$ and $A>0$. Define

$$
\begin{aligned}
& \tilde{\varphi}(x, \xi) \\
& =\iint \rho\left((x-y) \varphi(y, \eta)^{-1},(\xi-\eta) \Psi(y, \eta)^{-1}\right)(\varphi \Psi)(y, \eta)^{-n} \varphi(y, \eta) d y d \eta \\
& \widetilde{\Psi}(x, \xi) \\
& =\iint \rho\left((x-y) \varphi(y, \eta)^{-1},(\xi-\eta) \Psi(y, \eta)^{-1}\right)(\varphi \Psi)(y, \eta)^{-n} \Psi(y, \eta) d y d \eta \\
& T(x, \xi) \\
& =\iint \rho\left((x-y) \widetilde{\varphi}(x, \xi)^{-1},(\xi-\eta) \tilde{\Psi}(x, \xi)^{-1}\right)(\tilde{\varphi} \tilde{\Psi})(x, \xi)^{-n} \tilde{T}(y, \eta) d y d \eta
\end{aligned}
$$

where $\widetilde{T}(y, \eta)=\prod_{k=1}^{d} T_{k}(y, \eta)$.

Lemma 3.5. There is a positve constant $C$ such that (i) $C^{-1} \varphi(x, \xi) \leq \tilde{\varphi}(x, \xi) \leq$ $C \varphi(x, \xi), C^{-1} \Psi(x, \xi) \leq \widetilde{\Psi}(x, \xi) \leq C \Psi(x, \xi)$, and $C^{-1} \widetilde{T}(x, \xi) \leq T(x, \xi) \leq C \widetilde{T}(x, \xi)$ for $(x, \xi) \in \mathbf{R}^{2 n}$. (ii)

$$
\begin{aligned}
\left|\widetilde{\varphi}_{(\beta)}^{(\alpha)}(x, \xi)\right| / \widetilde{\varphi}(x, \xi)+\left|\tilde{\Psi}_{(\beta)}^{(\alpha)}(x, \xi)\right| / \tilde{\Psi}(x, \xi) & +\left|T_{(\beta)}^{(\alpha)}(x, \xi)\right| / T(x, \xi) \\
\leq & \leq C^{|\alpha+\beta|+1}|\alpha+\beta| !^{\kappa} \varphi(x, \xi)^{-|\beta|} \Psi(x, \xi)^{-|\alpha|}
\end{aligned}
$$

for $(x, \xi) \in \mathbf{R}^{2 n}$. (iii) $\widetilde{\varphi}$ and $\widetilde{\Psi}$ satisfy (i), (ii) and (iii) in Lemma 3.3, replacing $\varphi$ and $\Psi$ by $\tilde{\varphi}$ and $\tilde{\Psi}$, respectively.

We note that it follows from Lemma 3.4 and (i) of Lemma 3.5 that $T$ is $\sigma, g$ temperate. For $M>0$ we define

$$
\Lambda(x, \xi)=\Lambda(x, \xi ; M, N, h)=M \log T(x, \xi)
$$

where $T$ is given in (3.3). Denote

$$
\omega_{\beta}^{\alpha}(\Lambda ; x, \xi)=e^{-\Lambda(x, \xi)} D_{x}^{\beta} \partial_{\xi}^{\alpha} e^{\Lambda(x, \xi)}
$$

Then we have the following 
Lemma 3.6. There is a positive constant $C$, independent of $M$ and $N$, such that (i)

$$
\left|\Lambda_{(\beta)}^{(\alpha)}(x, \xi)\right| \leq M C^{|\alpha+\beta|+1}|\alpha+\beta|^{\kappa} \varphi(x, \xi)^{-|\beta|} \Psi(x, \xi)^{-|\alpha|}
$$

for $(x, \xi) \in \mathbf{R}^{2 n}$ and $|\alpha+\beta| \geq 1$, and (ii)

$$
\left|D_{x}^{\delta} \partial_{\xi}^{\gamma} \omega_{\beta}^{\alpha}(\Lambda ; x, \xi)\right| \leq C\left(C \varphi^{-1}\right)^{|\beta+\delta|}\left(C \Psi^{-1}\right)^{|\alpha+\gamma|} \sum_{j=0}^{|\alpha+\beta|}(C M)^{|\alpha+\beta|-j}(|\gamma+\delta|+j) !^{\kappa}
$$

for $(x, \xi) \in \mathbf{R}^{2 n}$.

We obtain from (ii) of Lemma 3.6

$$
\begin{aligned}
\left|D_{x}^{\delta} \partial_{\xi}^{\gamma} \omega_{\beta}^{\alpha}(\Lambda ; x, \xi)\right| \leq C^{|\alpha+\beta+\gamma+\delta|+1}|\gamma+\delta| !^{\kappa}\left(|\alpha+\beta|^{\kappa}+M\right)^{|\alpha+\beta|} & \\
& \times \varphi(x, \xi)^{-|\beta+\delta|} \Psi(x, \xi)^{-|\alpha+\gamma|} \text { for }(x, \xi) \in \mathbf{R}^{2 n}
\end{aligned}
$$

noting that $M^{|\alpha+\beta|-j}(|\gamma+\delta|+j) !^{\kappa} \leq C_{1}^{|\alpha+\beta|+|\gamma+\delta|}|\gamma+\delta| !^{\kappa}\left(j^{\kappa}+M\right)^{|\alpha+\beta|}$ for $j \leq|\alpha+\beta|$. Following [4], for a positive function $m(x, \xi)$ and the metric $g=\varphi^{-2}|d x|^{2}+\Psi^{-2}|d \xi|^{2}$ we define the symbol class $S(m, g)$ of pseudodifferential operators by the set of all $a(x, \xi) \in C^{\infty}\left(\mathbf{R}^{2 n}\right)$ satisfying

$$
\left|a_{(\beta)}^{(\alpha)}(x, \xi)\right| \leq C_{\alpha \beta} m(x, \xi) \varphi(x, \xi)^{-|\beta|} \Psi(x, \xi)^{-|\alpha|} \text { for }(x, \xi) \in \mathbf{R}^{2 n}
$$

Denote by $e^{\Lambda}(x, D)$ the pseudodifferential operator whose symbol is $e^{\Lambda(x, \xi)}$ and by $R e^{-\Lambda}(x, D)$ the reversed operator of $e^{-\Lambda}(x, D)$, i.e.,

$$
R e^{-\Lambda}(x, D) u(x)=(2 \pi)^{-n} \iint e^{i(x-y) \cdot \xi-\Lambda(y, \xi)} u(y) d y d \xi
$$

for $u \in C_{0}^{\infty}\left(\mathbf{R}^{n}\right)$. Put $q(x, D)={ }^{R} e^{-\Lambda}(x, D) e^{\Lambda}(x, D)$. Then we can express

$$
q(x, \xi)=(2 \pi)^{-n} o s-\iint e^{-i y \cdot \eta-\Lambda(x+y, \xi+\eta)+\Lambda(x+y, \xi)} d y d \eta
$$

where os- $\iint$ means an oscillatory integral. By Taylor's expansion we have

$$
D_{x}^{\beta} \partial_{\xi}^{\gamma} q(x, \xi)=\sum_{|\alpha|<\ell} D_{x}^{\alpha+\beta} \partial_{\xi}^{\gamma} \omega^{\alpha}(-\Lambda ; x, \xi) / \alpha !+D_{x}^{\beta} \partial_{\xi}^{\gamma} q_{\ell}(x, \xi)
$$


where $\omega^{\alpha}(-\Lambda ; x, \xi)=e^{\Lambda(x, \xi)} \partial_{\xi}^{\alpha} e^{-\Lambda(x, \xi)}$ and

$$
\begin{aligned}
& q_{\ell(\beta)}^{(\gamma)}(x, \xi) \equiv D_{x}^{\beta} \partial_{\xi}^{\gamma} q_{\ell}(x, \xi) \\
& =(2 \pi)^{-n} o s-\iint e^{-i y \cdot \eta} \sum_{|\alpha|=\ell} \alpha !^{-1} \\
& \quad \times \int_{0}^{1} \ell(1-\theta)^{\ell-1} D_{x}^{\alpha+\beta} \partial_{\xi}^{\gamma}\left\{e^{\Lambda_{1}}\left(\partial_{\xi}^{\alpha} e^{-\Lambda_{2}}\right)\right\} d y d \eta d \theta \\
& =(2 \pi)^{-n} o s-\iint e^{-i y \cdot \eta} \int_{0}^{1}(1-\theta)^{\ell-1} e^{\Lambda_{1}-\Lambda_{2}} \omega_{\ell \beta}^{\gamma}(x+y, \xi, \theta \eta) d y d \eta d \theta
\end{aligned}
$$

where $\Lambda_{1}=\Lambda(x+y, \xi), \Lambda_{2}=\Lambda(x+y, \xi+\theta \eta)$ and

$$
\begin{aligned}
& \omega_{\ell \beta}^{\gamma}(x+y, \xi, \theta \eta)=\ell \sum_{|\alpha|=\ell} \alpha !^{-1} e^{\Lambda_{2}-\Lambda_{1}} D_{x}^{\alpha+\beta} \partial_{\xi}^{\gamma}\left\{e^{\Lambda_{1}}\left(\partial_{\xi}^{\alpha} e^{-\Lambda_{2}}\right)\right\} . \\
& =\ell \sum_{|\alpha|=\ell} \sum_{\gamma^{\prime} \leq \gamma, \beta^{\prime} \leq \alpha+\beta} \alpha !^{-1}\left(\begin{array}{c}
\alpha+\beta \\
\alpha^{\prime}
\end{array}\right)\left(\begin{array}{c}
\gamma \\
\gamma^{\prime}
\end{array}\right) \\
& \quad \times \omega_{\beta^{\prime}}^{\gamma^{\prime}}(\Lambda ; x+y, \xi) \omega_{\alpha+\beta-\beta^{\prime}}^{\alpha+\gamma-\gamma^{\prime}}(-\Lambda ; x+y, \xi+\theta \eta) .
\end{aligned}
$$

Denote

$$
\begin{gathered}
g_{1}=g_{(x+y, \xi)}(0, \eta), \quad \varphi_{1}=\varphi(x+y, \xi), \quad \varphi_{2}=\varphi(x+y, \xi+\theta \eta), \\
\Psi_{1}=\Psi(x+y, \xi), \quad \Psi_{2}=\Psi(x+y, \xi+\theta \eta), \quad \omega_{\beta}^{\alpha}\left(\Lambda_{1}\right)=\omega_{\beta}^{\alpha}(\Lambda ; x+y, \xi), \\
\omega_{\beta}^{\alpha}\left(-\Lambda_{2}\right)=\omega_{\beta}^{\alpha}(-\Lambda ; x+y, \xi+\theta \eta) .
\end{gathered}
$$

Then we have the following

Lemma 3.7. There are $C>0$ and $c_{0}>0$, independent of $N$ and $M$, such that

$$
\begin{aligned}
& \left|D_{x, y}^{\delta} \partial_{\xi, \eta}^{\rho} \omega_{l \beta}^{\gamma}(x+y, \xi, \theta \eta)\right| \\
& \quad \leq C^{|\beta+\gamma|+|\delta+\rho|+1}(|\beta+\gamma|+|\delta+\rho|) !^{\kappa} \varphi_{1}^{-|\beta|-|\delta|} \Psi_{1}^{-|\gamma|-|\rho|}\left\{C M^{2 \kappa-1} N^{-1}\right\}^{\ell}
\end{aligned}
$$

for $\ell^{\prime}=M$ if $g_{1} \leq c_{0}$.

By virtue of (3.5), noting that $\varphi \Psi \geq c N$, we can estimate $\omega^{\alpha}(-\Lambda)$ in (3.6) as follows:

$$
\begin{aligned}
& \left|D_{x}^{\beta} \partial_{\xi}^{\gamma} D_{x}^{\alpha} \omega^{\alpha}(-\Lambda)\right| / \alpha ! \\
& \leq \alpha !^{-1} C^{|\gamma+\beta+\alpha|}|\gamma+\beta+\alpha| !^{\kappa}\left(|\alpha|^{\kappa}+M\right)^{|\alpha|} \varphi^{-|\beta+\alpha|} \Psi^{-|\alpha+\gamma|} \\
& \leq C_{1}^{\beta+\gamma+\alpha \mid}|\beta+\gamma| !^{\kappa}\left\{|\alpha|^{(2 \kappa-1)}+|\alpha|^{\kappa-1} M\right\}^{|\alpha|} \varphi^{-|\beta|} \Psi^{-|\gamma|} N^{-|\alpha|} \\
& \leq C_{1}^{\gamma+\beta \mid}|\gamma+\beta| !^{\kappa}\left\{C_{1} M^{2 \kappa-1} N^{-1}\right\}^{|\alpha|} \varphi^{-|\beta|} \Psi^{-|\gamma|}
\end{aligned}
$$


for $|\alpha| \leq \ell=M$. Let us prove that

$$
\left|q_{\ell(\beta)}^{(\cdot \alpha)}(x, \xi)\right| \leq C_{\alpha \beta}\left\{C M^{2 \kappa-1} N^{-1}\right\}^{\ell} \varphi^{-|\beta|} \Psi^{-|\alpha|}
$$

for $\ell=M$, where $C_{\alpha \beta}$ is independent of $M$ and $N$, and $C$ is independent of $M, N, \alpha$ and $\beta$. Let $\chi(t)$ be in $C_{0}^{\infty}(\mathbf{R})$ such that $\chi(t)=1$ for $|t| \leq 1 / 2$ and $\chi(t)=0$ for $|t| \geq 1$, and

$$
\left|D_{t}^{j} \chi(t)\right| \leq C A^{j} j !^{\kappa} \quad \text { for any } j \text { and } t \in \mathbf{R},
$$

where $A>0$. In brief we denote

$$
\begin{gathered}
z_{1}=(x+y, \xi), \quad z_{2}=(x+y, \xi+\theta \eta) \\
\varphi_{i}=\varphi\left(z_{i}\right), \quad \Psi_{i}=\Psi\left(z_{i}\right), \quad \tilde{\varphi}_{i}=\tilde{\varphi}\left(z_{i}\right), \quad \tilde{\Psi}_{i}=\tilde{\Psi}\left(z_{i}\right) \quad(i=1,2) \\
\chi_{1}=\chi\left(c_{0}^{-1} \tilde{\Psi}\left(z_{1}\right)^{-2}|\eta|^{2}\right) .
\end{gathered}
$$

Then we can write from (3.7)

$$
\begin{aligned}
& q_{\ell(\beta)}^{(\gamma)}(x, \xi)=(2 \pi)^{-n} \iiint_{0}^{1} e^{-i y \cdot \eta} \chi_{1}(1-\theta)^{\ell-1} e^{\Lambda_{1}-\Lambda_{2}} \omega_{\ell \beta}^{\gamma} d y d \eta d \theta \\
& \quad+(2 \pi)^{-n} \iiint_{0}^{1} e^{-i y \cdot \eta}\left(1-\chi_{1}\right)(1-\theta)^{\ell-1} e^{\Lambda_{1}-\Lambda_{2}} \omega_{\ell \beta}^{\gamma} d y d \eta d \theta \equiv I_{1}+I_{2},
\end{aligned}
$$

where $\omega_{\ell \beta}^{\gamma}=\omega_{\ell \beta}^{\gamma}(x+y, \xi, \theta \eta)$ is given in (3.8).

Lemma 3.8. There is $C>0$ such that

$$
\left|D_{y}^{\beta} \partial_{\eta}^{\alpha} \chi_{1}\right| \leq C^{|\alpha+\beta|+1}|\alpha+\beta|^{\kappa} \varphi_{1}^{-|\beta|} \Psi_{1}^{-|\alpha|} .
$$

Now we shall estimate $I_{1}$ in (3.12). Noting that $\varphi_{1} \sim \varphi_{2}, \Psi_{1} \sim \Psi_{2}, e^{\Lambda_{1}-\Lambda_{2}} \leq C^{M}$ and $|\eta| \leq\langle\xi\rangle_{h} / 2$ on supp $\chi_{1}$, we can estimate, by use of (3.5), (3.9) and (ii) of Lemma 3.3 ,

$$
\begin{aligned}
& \mid\left(\partial_{\eta}^{\rho}\left\{e^{\Lambda_{1}-\Lambda_{2}} \chi_{1} \omega_{\ell \beta}^{\gamma}(x+y, \xi, \theta \eta)\right\} \mid\right. \\
& \leq \sum_{\rho^{\prime}}\left(\begin{array}{c}
\rho \\
\rho^{\prime}
\end{array}\right)\left|\left(\partial_{\eta}^{\rho^{\prime}} e^{\Lambda_{1}-\Lambda_{2}}\right) \partial_{\eta}^{\rho-\rho^{\prime}}\left\{\chi_{1} \omega_{\ell \beta}^{\gamma}(x+y, \xi, \theta \eta)\right\}\right| \\
& \leq \sum_{\rho^{\prime}}\left(\begin{array}{c}
\rho \\
\rho^{\prime}
\end{array}\right) C^{M} C^{\left|\rho^{\prime}\right|}\left(\left|\rho^{\prime}\right|^{\kappa}+M\right)^{\left|\rho^{\prime}\right|} C^{\left|\rho-\rho^{\prime}+\beta+\gamma\right|}\left|\rho-\rho^{\prime}+\beta+\gamma\right| !^{\kappa} \\
& \quad \times\left(C M^{2 \kappa-1} N^{-1}\right)^{\ell} \varphi_{1}^{-|\beta|} \Psi_{1}^{-|\gamma+\rho|} \\
& \leq C_{1}^{|\rho+\beta+\gamma|}|\rho+\beta+\gamma|^{\kappa}\left(C_{1} M^{2 \kappa-1} N^{-1}\right)^{\ell} \varphi_{1}^{-|\beta|} \Psi_{1}^{-|\rho+\gamma|} \\
& \leq C_{\rho \beta \gamma}\left(C_{1} M^{2 \kappa-1} N^{-1}\right)^{\ell} \Psi_{1}^{-|\rho|} \varphi^{-|\beta|} \Psi^{-|\gamma|}\left(1+g_{(x+y, \xi)}^{\sigma}(y, 0)\right)^{|\beta+\gamma| / 2}
\end{aligned}
$$


for $\ell=M$. Since $g_{(x+y, \xi)}^{\sigma}(y, 0)=\Psi_{1}^{2}|y|^{2}$, we can estimate from (3.13)

$$
\begin{aligned}
\left|I_{1}\right|= & \mid(2 \pi)^{-n} \iiint_{0}^{1} e^{-i y \cdot \eta}(1-\theta)^{\ell-1}\left(1-\Psi_{1}^{2} \Delta_{\eta}\right)^{p} \\
& \times\left[\left(1+\Psi_{1}^{2}|y|^{2}\right)^{-p}\left\{\chi_{1} e^{\Lambda_{1}-\Lambda_{2}} \omega_{\ell \beta}^{\gamma}(x+y, \xi, \theta \eta)\right\}\right] d y d \eta d \theta \mid \\
\leq & C_{p \beta \gamma}\left\{C M^{2 \kappa-1} N^{-1}\right\}^{\ell} \varphi^{-|\beta|} \Psi^{-|\gamma|} \\
& \times \iiint_{\text {supp } \chi_{1}}\left(1+\Psi_{1}^{2}|y|^{2}\right)^{-p+|\beta+\gamma| / 2} d y d \eta d \theta
\end{aligned}
$$

for $p \geq|\beta+\gamma| / 2$. Since $|\eta| \leq C \Psi_{1}$ on supp $\chi_{1}$, taking account of Lemma 3.3 we get

$$
\begin{aligned}
& \iiint_{\text {supp } \chi_{1}}\left(1+\Psi_{1}^{2}|y|^{2}\right)^{-p+|\beta+\gamma| / 2} d y d \eta d \theta \\
& \leq C_{n} \int\left(1+\Psi_{1}^{2}|y|^{2}\right)^{-p+|\beta+\gamma| / 2} \Psi_{1}^{n} d y \\
& \leq C_{n p} \int\left\{1+\Psi^{2}|y|^{2}\left(1+N^{-2} \Psi^{2}|y|^{2}\right)^{-1}\right\}^{-p+|\beta+\gamma| / 2} \\
& \quad \times \Psi^{n}\left(1+N^{-2} \Psi^{2}|y|^{2}\right)^{n / 2} d y \\
& \leq C_{1} \int(1+\Psi|y|)^{-p+|\beta+\gamma| / 2+n} \Psi^{n} d y \leq C_{2},
\end{aligned}
$$

if we choose $p \geq|\beta+\gamma| / 2+2 n+1$. Thus we obain from (3.14)

$$
\left|I_{1}\right| \leq C_{\beta \gamma}\left(C M^{2 \kappa-1} N^{-1}\right)^{\ell} \varphi^{-|\beta|} \Psi^{-|\gamma|} \text { for } \ell=M
$$

Next we shall estimate $I_{2}$ in (3.12). From (3.7) and (3.8) we can write

$$
\begin{aligned}
& \left(1-\chi_{1}\right) e^{\Lambda_{1}-\Lambda_{2}} \omega_{\ell \beta}^{\gamma}=\ell \sum_{\alpha=\ell} \alpha !^{-1}\left(1-\chi_{1}\right)\left\{e^{\Lambda_{1}}\left(e^{-\Lambda_{2}}\right)^{(\alpha)}\right\}_{(\alpha+\beta)}^{(\gamma)} \\
& =\ell \sum_{|\alpha|=\ell} \alpha !^{-1} \sum_{\substack{\alpha=\ell \\
\beta^{\prime} \leq \alpha+\beta, \gamma^{\prime} \leq \gamma}}\left(\begin{array}{c}
\alpha+\beta \\
\beta^{\prime}
\end{array}\right)\left(\begin{array}{c}
\gamma \\
\gamma^{\prime}
\end{array}\right)\left(1-\chi_{1}\right)\left(e^{\Lambda_{1}}\right)_{\left(\beta^{\prime}\right)}^{\left(\gamma^{\prime}\right)} \\
& \quad \times\left(e^{-\Lambda_{2}}\right)_{\left(\begin{array}{c}
\left.\alpha+\gamma-\gamma^{\prime}\right) \\
\left(\alpha+\beta-\beta^{\prime}\right)
\end{array}\right.}
\end{aligned}
$$

where $w_{(\beta)}^{(\alpha)}$ denotes $D_{x}^{\beta} \partial_{\xi}^{\alpha} w(x, \xi)$. For simplicity we put

$$
r_{1}=\left(1-\chi_{1}\right)\left(e^{\Lambda_{1}}\right)_{\left(\beta^{\prime}\right)}^{\left(\gamma^{\prime}\right)}, \quad r_{2}=\left(e^{-\Lambda_{2}}\right)_{\left(\alpha+\beta-\beta^{\prime}\right)}^{\left(\alpha+\gamma-\gamma^{\prime}\right)}
$$

Then it follows from (3.5) and Lemma 3.8 that

$$
\left|D_{y}^{\delta} \partial_{\eta}^{\rho} r_{1}\right| \leq C_{\rho \beta^{\prime} \gamma^{\prime}} C^{M}\left\{C\left(|\delta|^{\kappa}+M\right)\right\}^{|\delta|} e^{\Lambda_{1}} \varphi_{1}^{-\left|\beta^{\prime}+\delta\right|} \Psi_{1}^{-\left|\gamma^{\prime}+\rho\right|}
$$


Similarly, we can estimate

$$
\left|D_{y}^{\delta} \partial_{\eta}^{\rho} r_{2}\right| \leq C_{\rho \beta \gamma} C^{M}\left\{C\left(|2 \alpha+\delta|^{\kappa}+M\right)\right\}^{|2 \alpha+\delta|} e^{-\Lambda_{2}} \varphi_{2}^{-\left|\alpha+\beta-\beta^{\prime}+\delta\right|} \Psi_{2}^{-\left|\alpha+\gamma-\gamma^{\prime}+\rho\right|}
$$

Therefore, we obtain from (3.15)-(3.17)

$$
\begin{aligned}
\left|I_{2}\right| & =\left|(2 \pi)^{-n} \iiint_{0}^{1} e^{-i y \cdot \eta}\left\{(1-\theta)^{\ell-1}\left(1-\chi_{1}\right)\right\} e^{\Lambda_{1}-\Lambda_{2}} \omega_{\ell \beta}^{\gamma} d y d \eta d \theta\right| \\
= & \left.\left|(2 \pi)^{-n} \iiint_{0}^{1} e^{-i y \cdot \eta}\right| \eta\right|^{-2 s} \Delta_{y}^{s}\left[\left\{(1-\theta)^{\ell-1}\left(1-\chi_{1}\right) e^{\Lambda_{1}-\Lambda_{2}}\right\}\right. \\
& \left.\times \omega_{\ell \beta}^{\gamma}\right] d y d \eta d \theta \mid \\
\leq & (2 \pi)^{-n} \ell \sum_{|\alpha|=\ell} \alpha !^{-1} \sum_{\beta^{\prime} \leq \alpha+\beta, \gamma^{\prime} \leq \gamma}\left(\begin{array}{c}
\alpha+\beta \\
\beta^{\prime}
\end{array}\right)\left(\begin{array}{c}
\gamma \\
\gamma^{\prime}
\end{array}\right) \sum_{|\delta|=s} s ! \delta !^{-1} \\
& \times\left.\sum_{\delta^{\prime} \leq 2 \delta}\left(\begin{array}{c}
2 \delta \\
\delta^{\prime}
\end{array}\right)\left|\iiint_{0}^{1} e^{-i y \cdot \eta}\left(1-\Psi_{1}^{2} \Delta_{\eta}\right)^{p}\left(1+\Psi_{1}^{2}|y|^{2}\right)^{-p}\right| \eta\right|^{-2 s} \\
& \times\left\{\left(1-\chi_{1}\right)\left(e^{\Lambda_{1}}\right)_{\left(\beta^{\prime}\right)}^{\left(\gamma^{\prime}\right)}\right\}_{\left(\delta^{\prime}\right)}\left\{\left(e^{-\Lambda_{2}}\right)_{\left(\alpha+\beta-\beta^{\prime}\right)}^{\left(\alpha+\gamma-\gamma^{\prime}\right)}\right\}_{\left(2 \delta-\delta^{\prime}\right)}(1-\theta)^{\ell-1} d y d \eta d \theta \mid .
\end{aligned}
$$

By (3.16) and (3.17), a simple calculation yields

$$
\begin{aligned}
& \left.\left|\left(1-\Psi_{1}^{2} \Delta_{\eta}\right)^{p}\left(1+\Psi_{1}^{2}|y|^{2}\right)^{-p}\right| \eta\right|^{-2 s} D_{y}^{\delta^{\prime}} r_{1} D_{y}^{2 \delta-\delta^{\prime}} r_{2} \mid \\
& \leq C_{p \beta \gamma} C^{M}\left\{C\left((|2 \alpha|+2 s)^{\kappa}+M\right)\right\}^{|2 \alpha|+2 s}\left(1+\Psi_{1}^{2}|y|^{2}\right)^{-p}\left(1+\Psi_{1} / \Psi_{2}\right)^{2 p} \\
& \quad \times e^{\Lambda_{1}-\Lambda_{2}}|\eta|^{-2 s} \varphi_{1}^{-\left|\beta^{\prime}+\delta^{\prime}\right|} \Psi_{1}^{-\left|\gamma^{\prime}\right|} \varphi_{2}^{-\left|\alpha+\beta-\beta^{\prime}+2 \delta-\delta^{\prime}\right|} \Psi_{2}^{-\left|\alpha+\gamma-\gamma^{\prime}\right|}
\end{aligned}
$$

where $\beta^{\prime} \leq \alpha+\beta, \gamma^{\prime} \leq \gamma, \delta^{\prime} \leq 2 \delta$ and $|\delta|=s$, since $|\eta|^{2} \geq c_{0} \Psi_{1}^{2} / 2$ on supp $\left(1-\chi_{1}\right)$. It follows from Lemma 3.4 that $e^{\Lambda_{1}-\Lambda_{2}} \leq\left\{C\left(1+N^{-2} g_{1}^{\sigma}\right)\right\}^{M d}$, where $g_{1}^{\sigma}=g_{z_{1}}^{\sigma}(0, \eta)$. Therefore, by Lemma 3.3 we have

$$
\begin{aligned}
e^{\Lambda_{1}-\Lambda_{2}} \varphi_{1}^{-\left|\beta^{\prime}+\delta^{\prime}\right|} \Psi_{1}^{-\left|\gamma^{\prime}\right|} \varphi_{2}^{-\left|\alpha+\beta-\beta^{\prime}+2 \delta-\delta^{\prime}\right|} \Psi_{2}^{-\left|\alpha+\gamma-\gamma^{\prime}\right|}\left(1+\Psi_{1} / \Psi_{2}\right)^{2 p} \\
\leq C^{\left|2 \alpha+\beta-\beta^{\prime}+2 \delta-\delta^{\prime}+\gamma-\gamma^{\prime}\right|+M d+p} \varphi_{1}^{-|\alpha+\beta|-2 s} \Psi_{1}^{-|\alpha+\gamma|} \\
\quad \times\left(1+N^{-2} g_{1}^{\sigma}\right)^{\left(\left|2 \alpha+\beta-\beta^{\prime}+\gamma-\gamma^{\prime}\right|+2 s\right) / 4+M d+p / 2} \\
\leq C_{p \beta \gamma} C_{1}^{s+M}\left\{1+N^{-2} g_{1}^{\sigma}\right\}^{(2 \ell+2 s+|\beta+\gamma|) / 4+M d+p / 2}\left(\varphi_{1} \Psi_{1}\right)^{-\ell} \varphi_{1}^{-2 s} \varphi^{-|\beta|} \Psi^{-|\gamma|} \\
\quad \times\left\{1+N^{-2} g_{(x+y, \xi)}^{\sigma}(y, 0)\right\}^{|\beta+\gamma| / 2},
\end{aligned}
$$

where $\beta^{\prime} \leq \alpha+\beta, \gamma^{\prime} \leq \gamma, \delta^{\prime} \leq 2 \delta,|\alpha|=\ell=M$ and $|\delta|=s$. Noting that

$$
g_{1}^{\sigma}=\varphi_{1}^{2}|\eta|^{2}=\left(\varphi_{1} \Psi_{1}\right)^{2} g_{1} \geq c_{0} d^{2} N^{2} / 2 \quad \text { for } g_{1} \geq c_{0} / 2
$$


we have by virtue of Lemma $\mathbf{3 . 3}$

$$
\begin{aligned}
\left|I_{2}\right| \leq & C_{p \beta \gamma} C_{2}^{s+M} M^{2 \kappa(s+M)} \iiint_{\operatorname{supp}\left(1-\chi_{1}\right)}\left(1+\Psi_{1}^{2}|y|^{2}\right)^{-p} \\
& \times\left(\varphi_{1}|\eta|\right)^{-2 s}\left\{N^{-2} g_{1}^{\sigma}\right\}^{(2 \ell+|\beta+\gamma|+2 s) / 4+M d p / 2} \\
& \times \varphi^{-|\beta|} \Psi^{-|\gamma|}\left(\varphi_{1} \Psi_{1}\right)^{-\ell}\left(1+\Psi_{1}^{2}|y|^{2}\right)^{|\beta+\gamma| / 2} d y d \eta d \theta \\
\leq & C_{p \beta \gamma} C_{3}^{\ell} N^{-(2 s-n-1)-\ell} M^{2 \kappa(s+M)} \varphi^{-|\beta|} \Psi^{-|\gamma|}
\end{aligned}
$$

if we choose $p \geq|\gamma+\beta| 2+n+1$ and $2 M d+p+|\gamma+\beta| / 2+\ell+n+1\} \leq s \leq C M+|\beta+\gamma|$. In fact, noting that $\left(1+\Psi_{1}|y|\right)^{-1} \leq C\left(1+\Psi_{1}|y|\right)(1+\Psi|y|)^{-1}$, Lemma 3.3 yields

$$
\begin{aligned}
& \iiint_{g_{1} \geq c_{0} / 2}\left(1+\Psi_{1}^{2}|y|^{2}\right)^{-p}\left(\varphi_{1}|\eta|\right)^{-(n+1)}\left(1+\Psi_{1}|y|\right)^{|\beta+\gamma|} d y d \eta d \theta \\
& \leq C_{\beta \gamma} \iiint(1+\dot{\Psi}|y|)^{-n-1}(1+\varphi|\eta|)^{-n-1}\left(1+\Psi_{1}|y|\right)^{-2 p+2 n+2+|\beta+\gamma|} d y d \eta \\
& \leq C_{\beta \gamma}^{\prime} \iint(1+\Psi|y|)^{-n-1}(1+\varphi|\eta|)^{-(n+1)} d y d \eta \leq C_{\beta \gamma}^{\prime \prime} .
\end{aligned}
$$

Choose $s, N$ and $\varepsilon$ so that

$$
\left\{\begin{array}{l}
N \geq M^{2-\varepsilon}, \quad 2-\varepsilon>\kappa \\
(2-\varepsilon)(2 s-n-1) \geq 2 \kappa s+M
\end{array}\right.
$$

Then we have

$$
\left|I_{2}\right| \leq C_{\beta \gamma}\left(C M^{2 \kappa-1} N^{-1}\right)^{\ell} \varphi^{-|\beta|} \Psi^{-|\gamma|}
$$

if

$$
1<\kappa<2
$$

Note that we can choose $\varepsilon>0$ so that $0<\varepsilon<2-\kappa$ if (3.19) is valid. Therefore, we can choose $s>0$ so that (3.18) is valid. Thus we have just proved (3.11). Moreover if $C M^{2 \kappa-1} N^{-1} \leq 1$, i.e., $0<\varepsilon<3-2 \kappa, 1<\kappa<3 / 2$ and $M \gg 1$, then from (3.6), (3.10) and (3.11) we have the following

Proposition 3.9. Assume $1<\kappa<3 / 2,0<\varepsilon<3-2 \kappa$ and $h \geq N \geq M^{2-\varepsilon} \gg 1$ are valid. Then $q(x, D)={ }^{R} e^{-\Lambda}(x, D) e^{\Lambda}(x, D)$ satisfies

$$
\left|\left\{q(x, \xi)-\sum_{|\alpha|<k} D_{x}^{\alpha} \omega^{\alpha}(-\Lambda ; x, \xi) \alpha !\right\}_{(\beta)}^{(\gamma)}\right| \leq C_{k \beta \gamma}\left(M^{2 \kappa-1} N^{-1}\right)^{k} \varphi^{-|\beta|} \Psi^{-|\gamma|}
$$


for any $k \geq 0$ with $k \leq M$, where $\omega^{\alpha}(-\Lambda ; x, \xi)=e^{\Lambda(x, \xi)} \partial_{\xi}^{\alpha} e^{-\Lambda(x, \xi)}$ and $C_{k \beta \gamma}$ is independent of $M, N$ and $h$.

Repeating the same argment as in Proposition 3.9, we can prove the following

Proposition 3.10. Assume that the same conditions in Proposition 3.9 are satisfied. Let $m(x, \xi)$ be a positive function defined in $T^{*}\left(\mathbf{R}^{n}\right)$ which is $\sigma, g$ temperate and $w(x, \xi) \in S(m, g)$ satisfying

$$
\left|w_{(\beta)}^{(\alpha)}(x, \xi)\right| \leq m(x, \xi) A^{|\alpha+\beta|}|\alpha+\beta|^{\kappa} \varphi^{-|\beta|} \Psi^{-|\alpha|} \quad \text { for }(x, \xi) \in \mathbf{R}^{2 n}
$$

Denote $k(x, \xi)=e^{\Lambda(x, \xi)} w(x, \xi)$ and $q(x, D)=R^{-\Lambda}(x, D) k(x, D)$. Then we have

$$
\begin{aligned}
& \left|\left\{q(x, \xi)-\sum_{|\alpha|<k} D^{\alpha}\left(\omega^{\alpha}(-\Lambda ; x, \xi) w(x, \xi)\right) / \alpha !\right\}_{(\beta)}^{(\gamma)}\right| \\
& \quad \leq C_{k \beta \gamma} m(x, \xi)\left(M^{2 \kappa-1} N^{-1}\right)^{k} \varphi^{-|\beta|} \Psi^{-|\gamma|} \quad \text { for any } k \text { with } k \leq M
\end{aligned}
$$

where $\omega^{\alpha}(\Lambda ; x, \xi)=e^{-\Lambda(x, \xi)} \partial_{\xi}^{\alpha} e^{\Lambda(x, \xi)}$ and $C_{k \beta \gamma}$ is independent of $M, N$ and $h$.

Now we can construct the inverse of $e^{\Lambda}(x, D)$. Put

$$
J(x, D)={ }^{R} e^{-\Lambda}(x, D) e^{\Lambda}(x, D)-I .
$$

Then it follows from Proposition 3.9 that $J(x, \xi) \in S(1, g)$ satisfies

$$
\|J(x, D)\|_{\mathcal{L}\left(L^{2}, L^{2}\right)} \leq C M^{2 \kappa-1} N^{-1} \leq C M^{2 \kappa-3+\varepsilon}<1
$$

if $\varepsilon<3-2 \kappa, 1<\kappa<3 / 2, N \geq M^{2-\varepsilon}$ and $M \gg 1$. Therefore $\{I+J(x, D)\}^{-1}=$ $\sum_{k=0}^{\infty}(-J(x, D))^{k}$ converges in $\mathcal{L}\left(L^{2}, L^{2}\right)$. Moreover it follows from Beals [2] that $(I+J(x, D))^{-1}$ is a pseudodifferential operator with symbol in $S(1, g)$. Thus we have obtained

$$
\left(e^{\Lambda}(x, D)\right)^{-1}=(I+J(x, D))^{-1 R} e^{-\Lambda}(x, D)
$$

which is also a pseudodifferntial operator with symbol in $S\left(C(M) e^{-\Lambda(x, \xi)}, g\right)$.

Let $g_{0}=\left(B \log \langle\xi\rangle_{h}\right)^{2}\left(|d x|^{2}+\langle\xi\rangle_{h}^{-2}|d \xi|^{2}\right)$ be a metric, where $B \geq 1$ is a parameter, and $g=\varphi^{-2}|d x|^{2}+\Psi^{-2}|d \xi|^{2}$ is the metric given in Lemma 3.3. In this case $H(x, \xi)=B \log \langle\xi\rangle_{h} \Psi(x, \xi)^{-1} \leq B d N^{-1} h^{-1 / 2} \log h \leq 1$ if $h^{1 / 2}(\log h)^{-1} \geq B$. Moreover $\left(g_{0}+g\right) / 2 \sim g$ and $g_{0} / g_{0}^{\sigma} \leq 1$ are valid. Therefore it follows from [4] that 
$\sigma(a(x, D) b(x, D))(x, \xi) \in S\left(m_{1} m_{2}, g\right)$ for $a \in S\left(m_{1}, g\right)$ and $b \in S\left(m_{2}, g_{0}\right)$, where $m_{1}$ and $m_{2}$ are $\sigma, g$ and $\sigma, g_{0}$ temperate, respectively.

For $p(x, \xi)$ in $S\left(\langle\xi\rangle_{h}^{m}, g_{0}\right)(m \in \mathbf{R})$ we define

$$
p_{\Lambda}(x, D)=\left(e^{\Lambda}(x, D)\right)^{-1} p(x, D) e^{\Lambda}(x, D)
$$

Then it follows from [4] that

$$
\sigma\left(p(x, D) e^{\Lambda}(x, D)\right)(x, \xi)-\sum_{|\alpha|<k} p^{(\alpha)}(x, \xi)\left(e^{\Lambda(x, \xi)}\right)_{(\alpha)} \alpha !^{-1}
$$

belongs to $S\left(C(M, k) e^{\Lambda(x, \xi)}\langle\xi\rangle_{h}^{m-k / 2}\left(B \log \langle\xi\rangle_{h}\right)^{k}, g\right)$, where $C(M, k)$ is independent of $h$ and $B$.

Lemma 3.11. Let $a(x, \xi)$ and $b(x, \xi)$ be in $S\left(m_{1}, g\right)$ and $S\left(m_{2}, g_{0}\right)$ respectively. Then

$$
a(x, \xi) b(x, \xi)-\sum_{|\alpha|<k}(-1)^{|\alpha|} \sigma\left(a^{(\alpha)}(x, D) b_{(\alpha)}(x, D)\right)(x, \xi) / \alpha !
$$

is in $S\left(m_{1} m_{2}\left(B \log \langle\xi\rangle_{h}\right)^{k}\langle\xi\rangle_{h}^{-k / 2}, g\right)$ for any integer $k \geq 0$.

Applying Lemma 3.11 to $\left(e^{\Lambda(x, \xi)}\right)_{(\alpha)} p^{(\alpha)}(x, \xi)$, we see that

$$
\begin{aligned}
& p^{(\alpha)}(x, \xi)\left(e^{\Lambda(x, \xi)}\right)_{(\alpha)} \\
& -\sum_{|\beta|<\ell}(-1)^{|\beta|} \sigma\left(k_{\alpha}^{(\beta)}(x, D) p_{(\beta)}^{(\alpha)}(x, D) / \beta !\right)(x, \xi)
\end{aligned}
$$

is in $S\left(C(M) e^{\Lambda} \Psi^{-|\alpha|}\langle\xi\rangle_{h}^{m-\ell / 2}\left(\log \langle\xi\rangle_{h}\right)^{\ell}, g\right)$, where $k_{\alpha}(x, \xi)=\left(e^{\Lambda(x, \xi)}\right)_{(\alpha)}$. Since $k_{\alpha}^{(\beta)}(x$, $\xi)=\partial_{\xi}^{\beta} D_{x}^{\alpha} e^{\Lambda(x, \xi)}=e^{\Lambda(x, \xi)} \omega_{\alpha}^{\beta}(\Lambda ; x, \xi)$, it follows from Proposition 3.10 and (3.5) that

$$
\sigma\left({ }^{R} e^{-\Lambda}(x, D) k_{\alpha}^{(\beta)}(x, D)\right)(x, \xi)-\sum_{|\gamma|<\ell} D_{x}^{\gamma}\left\{\omega^{\gamma}(-\Lambda ; x, \xi) \omega_{\alpha}^{\beta}(\Lambda ; x, \xi) / \gamma !\right\}
$$

is in $S\left(C(\ell,|\alpha+\beta|)\left(|\alpha+\beta|^{\kappa}+M\right)^{|\alpha+\beta|} \varphi^{-|\alpha|} \Psi^{-|\beta|}\left(M^{2 \kappa-1} N^{-1}\right)^{\ell}, g\right)$, for any $\ell$ with $\ell \leq M$, Moreover, noting that

$$
\sigma\left((I+J(x, D))^{-1}-I\right)=\sigma\left(-J(x, D)(I+J(x, D))^{-1}\right) \in S\left(M^{2 \kappa-1} N^{-1}, g\right),
$$

we get, from (3.20) and (3.24) with $\ell=1$,

$$
\sigma\left(\left(e^{\Lambda}(x, D)\right)^{-1} k_{\alpha}^{(\beta)}(x, D)\right)(x, \xi)-\omega_{\alpha}^{\beta}(\Lambda ; x, \xi)
$$


in $S\left(C_{\alpha \beta} M^{|\alpha+\beta|} \varphi^{-|\alpha|} \Psi^{-|\beta|} M^{2 \kappa-1} N^{-1}, g\right)$. From (3.22), (3.23) and (3.25) we have the following

Theorem 3.12. Assume that the same conditions in Proposition 3.9 are satisfied and, moreover, that $h^{1 / 2}(\log h)^{-1} \geq B \geq 1$ and $N \leq M^{2 \kappa}$. Let $p(x, \xi)$ be in $S\left(\langle\xi\rangle_{h}^{m}, g_{0}\right)$. Then for $p_{\Lambda}(x, D)$ given in $(3.21)$ we can see that

$$
p_{\Lambda}(x, \xi)-\sum_{|\alpha+\beta|<k} p_{(\beta)}^{(\alpha)}(x, \xi) w_{\alpha}^{\beta}(x, \xi)
$$

is in $S\left(C(M, k)\left(B \log \langle\xi\rangle_{h}\right)^{k}\langle\xi\rangle_{h}^{m-k / 2}, g\right)$ for any $k \geq 0$, where $w_{0}^{0}(x, \xi)=1$ and

$$
w_{\alpha}^{\beta}(x, \xi)-\left(-\Lambda_{\xi}(x, \xi)\right)^{\beta}\left(-i \Lambda_{x}(x, \xi)\right)^{\alpha} /(\alpha ! \beta !)
$$

is in $S\left(C_{\alpha \beta} M^{|\alpha+\beta|} \varphi^{-|\alpha|} \Psi^{-|\beta|} M^{2 \kappa-1} N^{-1}, g\right)$ for $|\alpha+\beta|>0$.

\section{Outline of the proof of Theorem 2.2}

In this section we shall give an outline of the proof of Theorem 2.2. Let $z^{0}=$ $\left(x^{0}, \xi^{0}\right) \in T^{*}\left(\mathbf{R}^{n}\right)$ with $\left|\xi^{0}\right|=1$ be a multiple characteristic point of $\tilde{p}$. We assume that $\widetilde{P}(x, D)$ satisfies (i), (ii) and (iii) of Proposition 2.1. For simplicity we denote $\widetilde{P}$ by $P$. Let $r$ be the multiplicity of $z^{0}$, i.e., $d^{j} p\left(z^{0}\right)=0$ for $j \leq r-1$ and $d^{r} p\left(z^{0}\right) \neq 0$. Following the notation in $\S 2$, we have

$$
\begin{aligned}
P(x, D ; \gamma) u(x) & =e^{-\gamma \zeta(x)} P(x, D)\left(e^{\gamma \zeta(x)} u(x)\right) \\
& =\sum_{|\alpha| \leq m} a_{\alpha}(x) \sum_{\alpha^{\prime} \leq \alpha}\left(\begin{array}{c}
\alpha \\
\alpha^{\prime}
\end{array}\right) \omega_{\alpha-\alpha^{\prime}}(\gamma \zeta) D_{x}^{\alpha^{\prime}} u(x)
\end{aligned}
$$

where we write $\omega_{\alpha}(\gamma \zeta)=e^{-\gamma \zeta} D_{x}^{\alpha} e^{\gamma \zeta}$. Note that $\omega_{\alpha}(\gamma \zeta)$ is a polynomial in $\gamma$ and its principal part is $\left(-i \gamma \zeta_{x}\right)^{\alpha}$. Therefore, we can write

$$
P(x, \xi ; \gamma)=\sum_{|\alpha| \leq m} P^{(\alpha)}(x, \xi) \omega_{\alpha}(\gamma \zeta) / \alpha !
$$

where $\omega_{\alpha}(\gamma \zeta)-\left(-i \gamma \zeta_{x}\right)^{\alpha}$ is a polynomial in $\gamma$ of degree $|\alpha|-1$ whose coefficients are in $\mathcal{B}^{\infty}\left(\mathbf{R}^{n}\right)$. Since $\Lambda_{+}(x, \xi)$ given in $\S 2$ satisfies

$$
\left|\Lambda_{+(\beta)}^{(\alpha)}(x, \xi)\right| \leq \begin{cases}C_{\alpha \beta}(a+|b|) \log \langle\xi\rangle_{h}\langle\xi\rangle_{h}^{-|\alpha|} & \text { for }(x, \xi) \in \mathbf{R}^{2 n} \\ C_{\alpha \beta}\left(a \log \langle\xi\rangle_{h}+|b|\right)\langle\xi\rangle_{h}^{-|\alpha|} & \text { if } \varphi_{2, h / 2}(x, \xi)=1 \text { and }|\alpha+\beta| \geq 1\end{cases}
$$


we have $e^{\Lambda_{+}(x, \xi)} \in S\left(e^{\Lambda_{+}}, g_{0}\right)$, where $g_{0}=\left\{(a+|b|) \log \langle\xi\rangle_{h}\right\}^{2}\left\{|d x|^{2}+\langle\xi\rangle_{h}^{-2}|d \xi|^{2}\right\}$. Moreover $\omega_{\alpha}^{\beta}\left(\Lambda_{+} ; x, \xi\right)=e^{-\Lambda_{+}(x, \xi)} D_{x}^{\beta} \partial_{\xi}^{\alpha} e^{\Lambda_{+}(x, \xi)}$ belongs to $S\left(C_{\alpha \beta}\left\{(a+|b|) \log \langle\xi\rangle_{h}\right\}^{|\alpha+\beta|}\right.$ $\left.\times\langle\xi\rangle_{h}^{-|\beta|}, g_{0}\right)$ and

$$
\omega_{\alpha}^{\beta}\left(\Lambda_{+} ; x, \xi\right)-\left(-\Lambda_{+\xi}\right)^{\alpha}\left(-i \Lambda_{+x}\right)^{\beta}
$$

is in $S\left(C_{\alpha \beta}\left\{(a+|b|) \log \langle\xi\rangle_{h}\right\}^{|\alpha+\beta|-1}\langle\xi\rangle_{h}^{-|\beta|}, g_{0}\right)$ for $|\alpha+\beta| \geq 1$. Put

$$
J_{+}(x, D)=e^{-\Lambda_{+}}(x, D) e^{\Lambda_{+}}(x, D)-I
$$

Then, $J_{+}(x, \xi)$ is in $S\left(\{(a+|b|) \log \langle\xi\rangle\}_{h}^{2}\langle\xi\rangle_{h}^{-1}, g_{0}\right)$. So we have $\left\|J_{+}(x, D)\right\|_{\mathcal{L}\left(L^{2}, L^{2}\right)}<1$ if $h \gg\{(a+|b|) \log h\}^{2}$ and the symbol of $\left(e^{\Lambda_{+}}(x, D)\right)^{-1}=\left(I+J_{+}(x, D)\right)^{-1} e^{-\Lambda_{+}}(x, D)$ belongs to $S\left(e^{-\Lambda_{+}}, g_{0}\right)$. On the other hand we have

$$
P\left(\Lambda_{+}, x, \xi ; \gamma\right)-\sum_{|\alpha+\beta|<\ell} P_{(\beta)}^{(\alpha)}(x, \xi ; \gamma) w_{+\alpha}^{\beta}(x, \xi)
$$

is in $S\left(C_{\ell}\langle\xi\rangle_{h}^{m}\left\{\langle\xi\rangle_{h}^{-1}(a+|b|)\left(\log \langle\xi\rangle_{h}\right)\right\}^{\ell}, g_{0}\right)$ for any $\ell \geq 0$ and $\gamma \leq h$, where $P\left(\Lambda_{+}, x, D\right.$; $\gamma)=e^{\Lambda_{+}}(x, D)^{-1} P(x, D ; \gamma) e^{\Lambda_{+}}(x, D)$ and

$$
w_{+\alpha}^{\beta}(x, \xi)-\left(-i \Lambda_{+x}\right)^{\alpha}\left(-\Lambda_{+\xi}\right)^{\beta} /(\alpha ! \beta !)
$$

is in $S\left(C_{\alpha \beta}\left\{(a+|b|) \log \langle\xi\rangle_{h}\right\}^{|\alpha+\beta|-1}\langle\xi\rangle_{h}^{-|\beta|}, g_{0}\right)$ for $|\alpha+\beta| \geq 1$ and $w_{+0}^{0}(x, \xi)=1$. Thus it follows from (4.1) and (4.2) that

$$
P\left(\Lambda_{+}, x, \xi ; \gamma\right)-\sum_{|\alpha+\beta|<\ell} P_{(\beta)}^{(\alpha)}(x, \xi) \widetilde{w}_{\alpha}^{\beta}(x, \xi, \gamma)
$$

is in $S\left(C_{\ell}\langle\xi\rangle_{h}^{m}\left\{\langle\xi\rangle_{h}^{-1}(a+|b|) \log \langle\xi\rangle_{h}\right\}^{\ell}, g_{0}\right)$ for any $\ell \geq 0$, where

$$
\widetilde{w}_{\alpha}^{\beta}(x, \xi, \gamma)-\left(-i \gamma \zeta_{x}-i \Lambda_{+x}\right)^{\alpha}\left(-\Lambda_{+\xi}\right)^{\beta} /(\alpha ! \beta !)
$$

is in $S\left(C_{\alpha \beta}\left\{\gamma+(a+|b|) \log \langle\xi\rangle_{h}\right\}^{|\alpha+\beta|-1}\langle\xi\rangle_{h}^{-|\beta|}, g_{0}\right)$ for $|\alpha+\beta| \geq 1\left(C_{\alpha \beta}\right.$ is independent of $\gamma$ and $h)$ and $\widetilde{w}_{0}^{0}(x, \xi, \gamma)=1$.

It suffices to show that $P\left(\Lambda_{+}, x, D ; \gamma\right)$ satisfies $(2.1)_{z^{0}}$. Then we can see that $P_{\Lambda_{+}}(x, D ; \gamma)=e^{-\Lambda_{+}}(x, D) P(x, D ; \gamma) e^{\Lambda_{+}}(x, D)$ also satisfies $(2.1)_{z^{0}}$

From now on we choose parameters $h, M, N, \gamma$, a and $b$ such that $h=\gamma$, $N=M^{2-\varepsilon} \geq d, h^{1 / 2} / \log h \geq a+|b|, a \geq 1$ and $b \in \mathbf{R}$, where $0<\varepsilon<3-2 \kappa$ 
and $1<\kappa<3 / 2$. Let $t_{k}(x, \xi)(k=1, \cdots, d)$ be the time functions of $p$ at $z^{0}$ which appear in $[\mathrm{A} . \mathrm{III}]_{z^{0}}$, and let $\Lambda(x, \xi)$ be defined by (3.4). We conjugate again $P\left(\Lambda_{+}\right)=P\left(\Lambda_{+}, x, D ; \gamma\right)$ by $e^{\Lambda}(x, D)$. Then it follows from (3.26), (3.27) of Theorem $3.12,(4.3)$ and (4.4) that

$$
P(\Lambda ; x, \xi)-\sum_{|\alpha+\beta|<\ell} P_{(\beta)}^{(\alpha)}(x, \xi) w_{\alpha}^{\beta}(x, \xi)
$$

is in $S\left(C(M, a, b)\langle\xi\rangle_{h}^{m-\ell / 2}\left(\log \langle\xi\rangle_{h}\right)^{\ell}, g\right)$ for any $\ell \geq 0$, where $P(\Lambda ; x, D)=\left(e^{\Lambda}(x, D)\right)^{-1}$ $\times P\left(\Lambda_{+}, x, D ; \gamma\right) e^{\Lambda}(x, D)$ and

$$
\rho_{\alpha}^{\beta}(x, \xi) \equiv w_{\alpha}^{\beta}(x, \xi)-\left(-i \gamma \zeta_{x}-i \Lambda_{+x}-i \Lambda_{x}\right)^{\alpha}\left(-\Lambda_{+\xi}-\Lambda_{\xi}\right)^{\beta} /(\alpha ! \beta !)
$$

is in $S\left(C_{\alpha \beta} M^{2 \kappa-1} N^{-1}\left[\left\{\gamma+(a+|b|) \log \langle\xi\rangle_{h}\right\} \varphi+M\right]^{|\alpha+\beta|} \varphi^{-|\alpha|} \Psi^{-|\beta|}, g\right)$.

We define the principal part of $P(\Lambda)=P(\Lambda ; x, D)$ by

$$
p(\Lambda ; x, \xi)=\sum_{|\alpha+\beta|<m+2} p_{(\beta)}^{(\alpha)}(x, \xi)\left(-i \gamma \zeta_{x}-i \Lambda_{+x}-i \Lambda_{x}\right)^{\alpha}\left(-\Lambda_{+\xi}-\Lambda_{\xi}\right)^{\beta} /(\alpha ! \beta !),
$$

where $p(x, \xi)$ is the principal part of $P(x, \xi)$. Then we can write from (4.5) and (4.6)

$$
\begin{aligned}
P(\Lambda ; x, \xi)=p(\Lambda ; x, \xi)+ & \sum_{0<|\alpha+\beta|<m+2} p_{(\beta)}^{(\alpha)}(x, \xi) \rho_{\alpha}^{\beta}(x, \xi) \\
& +\sum_{j=0}^{m-1} \sum_{|\alpha+\beta|<m+2} P_{j(\beta)}^{(\alpha)}(x, \xi) w_{\alpha}^{\beta}(x, \xi)+r(x, \xi),
\end{aligned}
$$

where $r(x, \xi) \in S\left(C(M, a, b)\langle\xi\rangle_{h}^{(m-2) / 2}\left(\log \langle\xi\rangle_{h}\right)^{m+2}, g\right)$ and $w_{\alpha}^{\beta}(x, \xi)$ and $\rho_{\alpha}^{\beta}(x, \xi)$ are given in (4.6).

For $\zeta=(y, \eta) \in \mathbf{R}^{2 n}$ we define

$$
p(x, \xi ; \zeta)=\sum_{|\alpha+\beta|<m+2} p_{(\beta)}^{(\alpha)}(x, \xi)(i \eta)^{\alpha}(-y)^{\beta} /(\alpha ! \beta !)
$$

Then we have

$$
p(\Lambda ; x, \xi)=p\left(x, \xi ; H_{\Lambda_{1}}\right)
$$

where $\Lambda_{1}=\gamma \zeta(x)+\Lambda_{+}+\Lambda$ and $H_{\Lambda_{1}}=\left(\Lambda_{1 \xi},-\Lambda_{1 x}\right)$. We put

$$
\begin{aligned}
Q(x, \xi)= & \left(\langle\xi\rangle_{h}^{2}\left|\Lambda_{1 \xi}\right|^{2}+\left|\Lambda_{1 x}\right|^{2}\right)^{-1 / 2} \\
& \times\left.\sum_{j=1}^{n}\left(\Lambda_{1 \xi_{j}} \partial / \partial x_{j}-\Lambda_{1 x_{j}} \partial / \partial \xi_{j}\right) p(x, \xi ; \zeta)\right|_{\zeta=H_{\Lambda_{1}}} \\
S(x, D)= & (2 i)^{-1}\left\{Q^{*}(x, D) P(\Lambda ; x, D)-P^{*}(\Lambda ; x, D) Q(x, D)\right\}
\end{aligned}
$$


where we denote by $Q^{*}$ the adjoint operator of $Q$ as $(Q u, v)_{L^{2}}=\left(u, Q^{*} v\right)_{L^{2}}$ for $u$ and $v \in C_{0}^{\infty}\left(\mathbf{R}^{n}\right)$. Taking $\psi_{h}(x, \xi)=\left(1-\Theta_{h}(\xi)\right) \psi(x, \xi)$, where $\psi \in C^{\infty}\left(T^{*}\left(\mathbf{R}^{n}\right)\right)$ is homogeneous in $\xi$ of degree 0 for $|\xi| \geq 1$ and $\psi=1$ in a conic neighborhood of $z^{0}$, we can prove that there are $c>0$ and $C>0$ such that

$$
\begin{aligned}
\left(S(\dot{x}, D) \psi_{h}(x, D) v, \psi_{h}(x, D) v\right)_{L^{2}} & \\
& \geq c\left\|\langle D\rangle_{h}^{(m-1) / 2} v\right\|_{L^{2}}^{2}-C\left\|\langle D\rangle_{h}^{m-1 / 2}\left(1-\psi_{h}\right) v\right\|_{L^{2}}^{2}
\end{aligned}
$$

for $v \in \mathcal{S}$. Then $(2.1)_{z^{0}}$ easily follows from (4.7). We omit the proof of (4.7), since the proof is not short and the method is similar to one used in [10]. For the proof of (4.7) we refer to [11].

\section{REFERENCES}

[1]. M.D. Bronshtein, Smoothness of polynomials depending on parameters, Sibirsk. Mat. Zh. 20 (1979), 493-509.

[2]. R. Beals, Weighted distribution spaces and pseudodifferential operators, J. d'analyse Math. 39 (1981), 131-187.

[3]. L. Gårding, Solution directe du problème de Cauchy pour les equations hyperboliques, Coll. Int. CNRS, Nancy, 1956, 71-90.

[4]. L. Hörmander, "The Analysis of Linear Partial Differential Operators III," Springer, Berlin-Heiderberg-New York-Tokyo, 1983.

[5]. V. Ja. Ivrii and V. M. Petkov, Necessary conditions for the Cauchy problem for non-strictly hyperbolic equations to be well posed, Uspehi Mat. Nauk 29 (1974), 3-68.

[6]. N. Iwasaki, The Cauchy problem for effectively hyperbolic equations (general case), J. Math. Kyoto Univ. 25 (1985), 727-743.

[7]. F. John, On linear partial differential equations with analytic coefficients, unique continuation of data, Comm. Pure Appl. Math. 2 (1949), 209-254.

[8]. K. Kajitani and T. Nishitani, "The Hyperbolic Cauchy Problem," Springer Lecture Note in Math. 1505, 1991.

[9]. K. Kajitani and S. Wakabayashi, Microlocal a priori estimates and the Cauchy problem $I$, to appear. 
[10]. K. Kajitani and S. Wakabayashi, Microlocal a priori estimates and the Cauchy problem II, to appear.

[11]. K. Kajitani, S. Wakabayashi and T. Nishitani, The Cauchy problem for hyperbolic operators of strong type, to appear.

[12]. J. Leray, "Hyperbolic Differential Equations," The Institute for Advanced Study, Princeton, 1953.

[13]. S. Mizohata, Some remarks on the Cauchy problem, J. Math. Kyoto Univ. 1 (1961), 109-127.

[14]. T. Nishitani, Hyperbolic operators with symplectic multiple characteristics, J. Math. Kyoto Univ. 29 (1989), 405-447.

[15]. T. Nishitani, Propagation of singularities for hyperbolic operators with transverse propagation cone, Osaka J. Math. 27 (1990), 1-16.

[16]. S. Wakabayashi, Singularities of solutions of the Cauchy problem for symmetric hyperbolic systems, Comm. in Partial Differential Equations 9 (1984), 1147-1177.

[17]. S. Wakabayashi, Generalized flows and their applications, Proc. NATO ASI on Advances in Microlocal Analysis, Series C, D. Reidel (1986), 363-384. 